\title{
A model for the jet-disk connection in BH accreting systems
}

\author{
A. Hujeirat ${ }^{1}$, M. Livio ${ }^{2}$, M. Camenzind ${ }^{3}$, and A. Burkert ${ }^{1}$ \\ 1 Max-Planck-Institut für Astronomie, 69117 Heidelberg, Germany \\ 2 SSTI, 3700 San Martin Drive, Baltimore, MD 21218, USA \\ ${ }^{3}$ Landessternwarte-Koenigstuhl, 69117 Heidelberg, Germany
}

Received 9 April 2003 / Accepted 30 June 2003

\begin{abstract}
The powerful and highly collimated jets observed in active galactic nuclei and $\mu$-quasars are likely to be connected to the accretion phenomenon via disks. Based on theoretical arguments and quasi-stationary radiative MHD calculations, a model for an accretion-powered jet is presented. It is argued that accretion disks around black holes consist of 1) a cold, Keplerian-rotating and weakly magnetized medium in the outer part, 2) a highly advective and turbulent-free plasma inside $r_{\text {tr }}=10-20$ Schwarzschild radii, where magnetic fields are predominantly of large scale topology and in excess of thermal equipartition, and 3) an ion-dominated torus in the vicinity of the hole, where magnetic fields undergo a topological change into a monopole like-configuration. The action of magnetic fields interior to $r_{\text {tr }}$ is to initiate torsional Alfvén waves that extract angular momentum from the disk-plasma and deposit it into the transition layer between the disk and the overlying corona, where the plasma is dissipative and tenuous. A significant fraction of the shear-generated toroidal magnetic field reconnects in the transition layer, thereby heating the plasma up to the virial-temperature and forming a super-Keplerian rotating, and hence centrifugally accelerated outflow.

The strong magnetic field in the transition layer forces the electrons to cool rapidly which, in combination with the fast outwardoriented motion, yields a two-temperature ion-dominated outflow. The toroidal magnetic field in the transition layer is in thermal equipartition with the ions, whereas the poloidal component is in equipartition with the electrons. Such a strong toroidal magnetic field is essential for increasing the jet-disk luminosity in the radio regime. These gravitationally unbound outflows serve as seeds, possibly, for all the powerful electron-proton jets observed in accreting systems containing black holes.
\end{abstract}

Key words. methods: $N$-body simulations - galaxies: jets - black hole physics - accretion, accretion disks - magnetohydrodynamics - radiative transfer

\section{Introduction}

Based on observational data, most of the systems containing jets are considered to be accreting systems, most likely with an accretion disk surrounding the central object. The most powerful and highly collimated jets are found to emanate from accreting systems containing, probably, black holes, with a maximum power attainable if the central objects are Kerr black holes (BHs) rotating at their maximum rate (Blandford 2001). Beside the numerous extragalactic radio sources with high gamma-factors $(\gamma \geq 3)$, the underlying engines powering the jets in microquasars such as GRS $1915+105$ and GRO 1655-40 are believed to be spinning BHs (Mirabel 2001).

Although there has been a significant progress in recent years toward understanding the morphology, propagation and shock diagnostics of jet-plasmas, no consensus has been reached yet about their basic driving mechanisms. Nevertheless, there are at least three ingredients that appear to be necessary for initiating jets that gained theoretical and

Send offprint requests to: A. Hujeirat, e-mail: hujeirat@mpia-hd.mpg.de observational supports: 1) large scale magnetic fields (MFs), 2) an accretion disk and 3) a central object which dominates the disk dynamics gravitationally. Magnetic fields in particular are considered to play a major role in the evolution of jets. The fact that many jets have comparable radio and bolometric luminosities hints to a significant fraction of the magnetic energy in their total power.

The aim of this paper is to present a model for jet formation from an accretion disk surrounding a Shwarzschild black hole. The model relies on accretion disks as primary sources of energies for powering jets. In particular, the innermost part of the disk, i.e., within 20 last stable radii, is the region where jets gain their vast rotational energy through magnetic braking. In order to describe the model in details, its differences and overlapping with other models, we briefly review several scenarios for initiating jets.

Indeed, several models have been suggested for the formation of jets (Ghosh \& Lamb 1987; Blandford \& Payne 1982; Pudritz \& Norman 1983; Uchida \& Shibata 1985; Shu et al. 1988; Königl 1989; Heyvearts \& Norman 1989; Camenzind 1990; Lovelace et al. 1991; Shu et al. 1994; Camenzind 2003; 
Hujeirat et al. 2002b). In the following we outline several properties of a few of these models:

1. The Blandford \& Payne model (1982, henceforth BP82) relies on magnetic extraction of angular momentum and rotational energy from an underlying cold and Keplerian disk, i.e., from a standard disk (SSD, Shakura \& Sunyaev 1973). Extraction is most efficient if the poloidal magnetic field is of a large scale topology and appropriately aligned to the disk-normal (i.e., the angle from disk-normal $\theta_{\mathrm{B}}$ must be larger than $30^{\circ}$ ). The second important result is that, at large radii, collimation is achieved through the action of the shear-generated toroidal MF. The difficulties of this model are:

(a) The disk is infinitely thin. This implies that Lorentz forces exerted on the disk-plasma would force the inflow to rotate sub-Keplerian, making it difficult for the plasma to be flung out by purely centrifugal means (Shu 1991). Furthermore, taking into account that toroidal magnetic field component $B_{\mathrm{T}}(z=0)=0$ and adopting the density profile $\rho \sim r^{-3 / 2}$ as BP82 suggested, any dynamo cycle will not succeed to amplify $B_{\mathrm{p}}$ at $z=0$ to values beyond thermal equipartition. One possible way to obtain ${ }^{1} \beta>1$ is through a collapse of the central part of the disk while freezing its magnetic flux. In this case ${ }^{2} B_{\mathrm{p}} \sim r^{-2}$, which implies that if $B_{\mathrm{p}}$ does not change topology, accretion will be terminated and the whole inflow will turn into outflow with unacceptably large mass load.

(b) It has been argued that even if the poloidal MFs intersects the disk with $\theta_{\mathrm{B}}>30^{\circ}$, thermal assistance is still required for the disk-plasma to overcome the potential difference parallel to the MF-lines.

(c) There is no special treatment to the flow in the deep gravitational well of the central object, which is necessary for increasing the total energy per gram of the inflow, and convert it into outflows with high speeds.

(d) The model relies on ideal MHD treatment to a cold Keplerian disk. However, since $\beta \geq 1$, MFs will likely suppress the generation and dissipation of turbulence. Hence, the disk is likely to be thermal unstable. If the accretion rate is high, the disk becomes optically thick to synchrotron/cyclotron radiation; gyrating electrons emit radiation on the cyclotron frequency, that in turn repeatedly upscattered by the hot electrons, establishing thereby a thermal equilibrium. Since this occurs on a time scale comparable or even shorter than the dynamical one, and in the absence of other efficient heating sources, the disk undergoes a runaway cooling: the temperature decreases and reach the lower limit $T_{\text {min }}=h v_{\text {peak }} / k$, where $v_{\text {peak }}$ is the peak cyclotron frequency. This thermally induced collapse of the inner disk gains acceleration if the accretion rate is low. Here the low frequency photons emitted by the gyrating electrons escape from the system without being absorbed or

\footnotetext{
${ }^{1} \beta=P_{\text {mag }} / P_{\text {gas }}$, where $P_{\text {mag }}=\left(B_{\mathrm{P}}^{2}+B_{\mathrm{T}}^{2}\right) / 4 \pi$, see Eq. (8) for further details.

${ }^{2} \Phi=2 \pi r^{2} B_{\mathrm{p}} \approx 2 \pi r^{2} B_{\theta}=$ const.
}

scattered, and so no lower limit for the temperature can be constructed.

2. The X-wind model (Shu et al. 1994). Here the magnetically driven-wind emanate, neither from the disk nor from the central object, but from the region around the co-rotation radius, $r_{\text {cor }}$, where the effective gravity vanishes. The model relies primarily on the poloidal MFs of the central object which is surrounded by an accretion disk, essentially without large scale magnetic fields. This approach relaxes the winding up problem of the poloidal MFs, which has been encountered in the Ghosh \& Lamb model (1987). Moreover, as outflows here originate from the region close to the surface of the star, their propagation speed is estimated to be of the order of the escape velocity.

when extending this model to accretion flows around BHs, the following problems arise:

(a) It is not applicable to accretion flows onto Schwarzschild BHs, as these objects do not possess dynamically stable poloidal MFs. If such holes are viewed as extreme stars, then the $\mathrm{X}$-wind model rule out indefinitely the possibility of jet initiation from weakly magnetized slowly rotating objects.

(b) Balbus \& Hawley instability amplifies weak MFs up to approximately thermal equipartition on the dynamical time scale. In the innermost region of the disk, MFs of the central object are beyond super-equipartition with respect to the thermal energy of the disk-plasma; generation and dissipation of turbulence will be suppressed, the plasma in the X-region (see Fig. 2b in Shu et al. 1994) becomes cold, and the radial accretion will be terminated.

(c) Almost all young stellar objects have been observed to rotate far below the beak-up velocity. Although the FU Orinois objects accrete at relatively high rates, they are considered to be slow rotators. Fast rotators, however, show excessive magnetic activities, which is in line with the Parker instability (Simon 1999). In the $\mathrm{X}$-wind model however, MFs in the X-region are nearly in equipartition with the potential energy of the flow. Such strong magnetic tubes are likely to be gravitationally unbound, cannot be anchored deep in the star and therefore they float upwards to the surface on the dynamical time scale. This occurs if they are generated in the convection zone or in the overshoot layer between the convection zone and the underlying rigidly rotating core, similar to the solar dynamo (Spiegel \& Zahn 1992).

3. Advection-dominated inflow outflow solutions - ADIOS (Blandford \& Begelman 1999),

ADIOS are special case of ADAF solutions (advected dominated accretion flows) in which the accretion rate is allowed to adopt the self-similar profile $\dot{\mathcal{M}} \sim r^{p}$, where $0 \leq p \leq 1$. Thus, the accretion rate decreases inwards, thereby giving rise to a substantial outflow with large mass load. ADIOS model is another step closer than ADAF toward explaining the low luminosity AGNs. Indeed, there are several numerical calculations that confirm the inward decrease of the accretion rate (e.g., Stone \& Pringle 2000; 
Hawley \& Balbus 2002). However, these calculations did not rule out the possibility that the outflows obtained might be large scale circulation. On the other hand, in addition to the difficulties associated with ADAF solutions, ADIOS model:

(a) Relies on one dimensional treatment to an intrinsically multi-dimensional phenomenon. The model does not provide answers to numerous questions related to jetmorphologies, such as the origin of the high speed of jet-propagation, the role of the central object, the magnetic field topology appropriate for initiating jets and etc.

(b) It largely overestimates the mass load of jets. Basically, it predicts that almost all accreted matter through the disk at large radii will re-appear in the jet. When applied to the galactic center, an accretion rate that is about one order of magnitude larger than the upper limit would be necessary to obtain a partial fitting with the observed SED (Yuan et al. 2002). Furthermore, neither ADAF nor ADIOS models are able to reproduce reasonable SED-fitting with observations in the radio frequency regime. They seriously underestimate the radio luminosities in AGNs and black hole X-ray transients (Esin et al. 2001; Di Matteo et al. 2003).

(c) The dynamical range of ADIOS is largely overestimated. To clarify this point, let $r_{\text {tr }}$ be the transition radius, at which an SSD makes a transition to ADAF (Fig. 2). If both solutions are thermally and dynamically stable, why should an SSD change into ADAF at large radii, or vice versa?

Mathematically, in the absence of external forces and for given Dirichlect boundary conditions, there is at most a single weak solution to the set of the corresponding linear systems of equations, which fulfills the entropy principle. Accordingly, at large radii the system of equations are nearly linear (i.e., they can be linearized as there are no mechanisms for generating shocks or strong gradients), and the conditions imposed at the outer boundary determine the solution uniquely. Note that the equations describing accretion flows in SSD or ADAF are predominantly Eulerian, except the angular momentum and energy equations that contain second order partial differential operators ${ }^{3}$. For example, if we set the angular velocity at the outer boundary to be subKeplerian and replacing $L_{\mathrm{E}}$ by $\Lambda_{\mathrm{B}}$ (see Eq. (12)), the SSD changes to ADAF. However, since there is no reason to expect an excessive source of heating at large radii, as in ADAF case, only cold standard disks are likely to survive there. These disks continue to maintain their thin geometrical structures all the way down to the central object, where other effects come into play, e.g., heat conduction, change of the MF-topology, noticeable

\footnotetext{
${ }^{3}$ In spherical geometry they read $L_{\mathrm{E}}=\nabla \cdot \lambda_{\mathrm{FLD}} \nabla E$, and $L_{\ell}=$ $\nabla \cdot \eta_{\text {tur }} \nabla \Omega$, respectively. $E$ and $\lambda_{\text {FLD }}$ are the radiation density and the radiative diffusion coefficient as defined in Eq. (12). $\Omega$ and $\eta_{\text {tur }}$ are the angular frequency and the coefficient of turbulent diffusion, respectively.
}

change of rotation-law and manifestation of the magnetic field and the gravitational well of the central object. Indeed, Abramowicz et al. (1998) concluded that a transition from SSD to ADAF may occur in the vicinity of the BH. Hujeirat \& Camenzind (2000a) found that the transition is thermally unstable, and the plasma in the outermost part of ADAF collapses dynamically, thereby extending the SSD down to several last-stable radii, where the disk truncates and an ion-dominated and highly edvective torus emerges.

An additional evidence that rules out transitions at large radii are MFs. ADAF/ADIOS models assume that MFs are in equipartition with the thermal energy. If this is the case, then this should apply also when MFs are of large scale topology. However, since the accretion flow is fully ionized and freely falling, magnetic flux freezing implies that the MF-strength will strongly increase inwards and become in equipartition with the potential energy of the flow, hence terminating accretion. This impose a constrain on the location of the transition radius: $r_{\text {tr }}$ must be smaller than 10-20 last-stable radii, depending on the accretion rate.

4. Truncated disks - advective tori solution (TDAT, Hujeirat $\&$ Camenzind 2000b). The model relies on the results of radiative HD calculations of two-temperature accretion flows onto BHs without magnetic fields. The important results of TDAT, that are relevant for jet-formation, read: 1) the disk truncates close to the last stable orbit and forms an iondominated and highly advective torus, 2) outward-oriented motions of plasmas between the disk and the overlying corona are formed, and that appear to be manifested when MFs are included (Hujeirat et al. 2002b).

Our approach in the present paper relies on theoretical arguments supported by the radiative two-temperature MHD numerical calculations. The model is based on the following assumptions: 1) the Balbus-Hawley instability acts as dynamo that amplifies the unordered MFs up to thermal equipartition, 2) the Parker- and BH-instabilities in combination with reconnection close a dynamo cycle, through which a large scale MF is generated, in the manner that Tout \& Pringle (1992) suggested. Once the large scale magnetic field grows beyond thermal equipartition, the generation and dissipation of turbulence will be suppressed. 3) A significant fraction of the toroidal magnetic field (TMF) lines re-connects in the transition layer (TL): a process which we treat by adopting a turbulent diffusivity $\eta_{\mathrm{mag}}$, 4) reconnection heats the plasma in the TL up to the virial temperature. The strong TMFs in the TL force the electrons to cool rapidly, yielding thereby a two-temperature ion-dominated outflow. Figure 2 shows the main evolutionary phases of our model for forming accretionpowered jets.

The paper runs as follows. In Sect. 3 we discuss the structure of magnetized accretion disks. The governing equations and the method of solution are described in Sect. 4. In Sect. 5 we present numerical results, which appear to be well described by self-similar solutions for the radiative MHD equations. We end up with Sect. 6 where the results are summarized. 


\section{Disk structure and magnetic braking}

\subsection{The outer part}

In SSDs the inward-advection of angular momentum is balanced by outward viscous transport. This is equivalent to equalize the terms $T 0$ and $T 3$ in Eq. (1), while all other terms are neglected

$$
\begin{aligned}
& \frac{\partial \ell}{\partial t}+\overbrace{\nabla \cdot V \ell}^{T 0}=\overbrace{B_{r} \frac{\partial r B_{\mathrm{T}}}{\partial r}}^{T 1}+\overbrace{B_{\theta} \frac{\partial B_{\mathrm{T}}}{\partial \theta}}^{T 2}+\overbrace{\frac{1}{r^{2} \frac{\partial}{\partial r} r^{4} \eta_{\mathrm{tur}} \frac{\partial \Omega}{\partial r}}}^{T 3} \\
& +\overbrace{\frac{1}{\cos \theta} \frac{\partial}{\partial \theta} \cos \theta \eta_{\mathrm{tur}} \frac{\partial \Omega}{\partial \theta}}^{T 4},
\end{aligned}
$$

where $\ell=r^{2} \sin \theta \Omega, \eta_{\text {tur }}=\rho v_{\text {tur }}$ is the turbulent viscosity coefficient (Shakura \& Sunyaev 1973), $V$ is the velocity field and $B$ is the magnetic field (see Eq. (8)). T0 denotes angular momentum transport via advection, $T 1$ and $T 2$ are for magnetic extraction, and $T 3$ and $T 4$ are for viscous (micro- or macroscopic hydrodynamical turbulence) re-distribution of angular momentum.

The key question here is how the constellation of these terms would look like in the vicinity of the black hole.

In the outermost regions of a disk, we anticipate magnetic fields to be below equipartition. Let $r_{\mathrm{tr}}$ be a transition radius (see Fig. 2), such that for $r>r_{\mathrm{tr}}$, we have the usual SSDs, where the ratio of magnetic to gas pressure is $\beta \ll 1$. Here the Balbus-Hawley instability (Balbus \& Hawley 1991) operates on the dynamical time scale: it rapidly amplifies the MF and forces $\beta$ to approach, but remains below, unity (Hawley et al. 1996). On the other hand, the rotational energy in SSDs exceeds the thermal energy by at least one order of magnitude. Therefore, the generated toroidal magnetic energy via shear can easily exceed thermal equipartition. Consequently, unless the imposed boundary conditions limit the amplification of the TMF, there is no reason to expect $\beta$ to remain strictly below unity.

\subsection{The inner part}

Whether the disk is surrounding compact object or a YSO, the innermost part of the disk is likely to be strongly magnetized. The question which then arise is: how does accretion onto BHs proceed under $\beta \geq 1$ condition?

In general, accretion via strongly magnetized and turbulence-free disks proceeds if the MFs are able to extract angular momentum from the rotating disk-plasma. The efficiency of this extraction depends, among others, on the MF-topology. For example, large scale or dipolar MF-topologies are considered to be appropriate for extracting rotational energy from the disk or even from the hole itself and power jets. In the present study, MFs are assumed to be of large scale topology. This is reasonable, as the $\mathrm{BH}$-instability in combination with the Parker instability may establish a dynamo cycle in which the inward-advected and initially weak MFs are amplified and then reconnect to build up the desired MF-configuration
(Tout \& Pringle 1992). A straightforwards conclusion that can be drawn is that when the matter at the outer region has completed one revolution around the central $\mathrm{BH}$, MFs should have reached equipartition almost everywhere in the disk. This would eventually suppress self-generated turbulence and terminating angular momentum transport through turbulencefriction.

In the region $r<r_{\mathrm{tr}}$, global conservation of the poloidal magnetic flux implies that $B_{\mathrm{P}} \sim r^{-2}$. Therefore, as the innermost part of the disk contracts, there is a critical radius $r_{\text {top }}$, below which gravitational-equipartition is maintained (i.e., Lorentz forces are of the same order as the gravitational forces), eventually terminating accretion. This configuration is unlikely, as this would imply that most of accretion inflow would be redirected into outflow, with an unacceptably large mass load. On the other hand, if $r_{\text {top }}$ is sufficiently close to the last stable orbit, a change of the MF-topology associated with a significant loss of magnetic flux through the horizon would be possible. This is a plausible event, as MFs close to the horizon are unlikely to adopt other than a monopole like-configuration (see Fig. 2b). Under such circumstances, the generation of $B_{\mathrm{T}}$ will be considerably reduced and the accretion of matter within $r_{\text {top }}$ proceeds unhindered.

Around the radius $r_{\text {top }}$ angular momentum transport is mediated through magnetic braking. When combining Eq. (1) with the equation corresponding to the time-evolution of the TMF (see Eq. (8)), and assuming the flow to be locally incompressible, we obtain a magnetic torsional wave equation which has the approximate form:

$\frac{\partial^{2}}{\partial^{2} t} B_{\mathrm{T}} \cong V_{\mathrm{A}}^{2} \Delta B_{\mathrm{T}}$.

$\Delta$ denotes the two-dimensional Poisson operator in spherical geometry, and $V_{\mathrm{A}}\left(\doteq B_{\mathrm{p}} / \sqrt{\rho}\right)$ is the Alfvén speed. The action of these waves is to magnetic-brakes the innermost part of the disk through transporting angular momentum from the disk to higher latitudes (see Figs. 1 and 4). Note that $B_{\mathrm{p}}$ determines uniquely the speed of propagation, hence the efficiency-dependence of angular momentum transport on the $B_{\mathrm{p}}$-topology. These torsional Alfvén waves (TAWs) propagate in the vertical direction on the time scale:

$\tau_{\mathrm{TAW}} \sim \frac{H}{V_{\mathrm{A}}} \sim \tau_{\text {dyn }}$.

This should be similar to the removal time scale of angular momentum from the disk ${ }^{4}$ :

$\tau_{\text {rem }} \sim \rho V_{\mathrm{T}} H / B_{\mathrm{P}} B_{\mathrm{T}} \sim r^{3 / 2}$.

Thus, $\tau_{\text {rem }}$ increases non-linearly with radius, attaining a minimum value at the inner boundary. This implies, for example, that the rate at which angular momentum is removed at the last stable orbit ( $\doteq R_{\mathrm{LSO}}$ ) is one thousand times faster than at $r=100 R_{\mathrm{LSO}}$. To avoid the innermost region of the disk from running out of angular momentum, we require the disk to be dynamically stable. Equivalently, the rate at which angular momentum is removed at any radius must be equal to the rate at

\footnotetext{
${ }^{4} \tau_{\text {rem }}$ is obtained from the terms $T 0$ and $T 2$ of Eq. (1).
} 


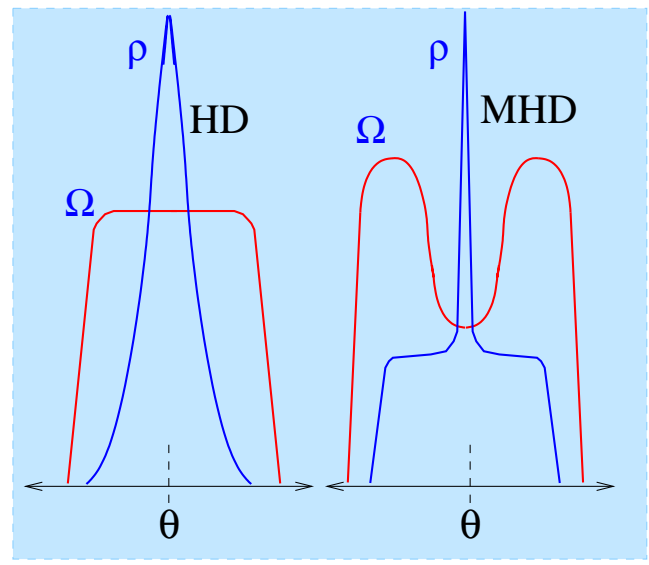

Fig. 1. A schematic distribution of the rotational velocity $\Omega$ and density $\rho$ across the disk with magnetic fields (MHD) and without (HD). The action of the equipartition MFs is to transport angular momentum from the disk to higher latitudes, where the particles are centrifugalaccelerated outwards.

which it is advected inwards from outer layers, i.e., $\tau_{\text {adv }}=\tau_{\text {rem. }}$. This implies that the radial velocity $-U$ in the disk is of the order of the Alfven speed, which is also of the order or even larger than the sound speed $V_{\mathrm{S}}$, hence the terminology advectiondominated disk.

The question which arises here is whether the disk, under these circumstances, maintains its geometrical thin structure.

The answer is hidden in the vertical momentum and energy equations. The former reads:

$\frac{\partial V}{\partial t}+V \cdot \nabla V=\frac{1}{\rho r} \frac{\partial P}{\partial \theta}+\tan \theta \frac{V_{\varphi}^{2}}{r}+\left.\frac{\nabla \times \nabla \times B}{4 \pi \rho}\right|_{\theta}$.

Taking into account that vertical transport of angular momentum is maintained through magnetic braking nearly without particle-advection (i.e., we neglect the second term in the LHS of the equation), and noting that $(\partial P / \partial \theta) / r \rho$ is the only term that opposes the vertical contraction of the disk, we obtain:

$\frac{H}{r} \approx \frac{V_{\mathrm{S}}}{V_{\varphi}}$.

This implies that, as in SSDs, the thickness of the disk depends strongly on the temperature of the plasma, and hence on the associated heating mechanisms. On the other hand, since $\beta \geq 1$ in the innermost part of the disk, the dominant source of heating in SSDs (i.e., turbulence dissipation), is no longer efficient. In this case, heating is mainly due to adiabatic compression and to other non-local sources, e.g., radiative reflection, Comptonization and conduction of heat flux from the surrounding media. However, these mechanisms are unlikely to change the disk configuration significantly, so that the disk continues to maintain its thin geometrical structure. Specifically, for $r \leq r_{\mathrm{tr}}$, we have $\partial H_{\mathrm{d}} / \partial r \geq 0$.

\subsection{Formation of the ion-dominated torus}

Whether the disk model is an SSD, ADAF or ADIOS, most of the physical variable (e.g., density temperature, radial and angular velocities) increase with decreasing radius. On the other hand, the disk is said to be truncated if within a certain radius, say the radius of the boundary layer $r_{\mathrm{BL}}$, part of the main physical variable start to decrease inwards. This occurs, for example, if the central object rotates sub-Keplerian ${ }^{5}$. The Kepler-rotating and inflowing material from the disk must release a significant fraction of its angular momentum to be able to cross the event horizon of a Schwarzschild BH. This might be achieved if the MFs in the BL are sufficiently strong to extract angular momentum from the disk on the dynamical time scale. However, this requires the density to decrease considerably inwards, so that the speed of propagation of the TAWs largely exceeds that in the outer disk.

We note that, unlike SSD and ADAF-models that predict a density-decrease with radius (i.e, $\rho \sim r^{-15 / 8}$ and $\rho \sim r^{-3 / 2}$, respectively), hydro- and MHD-calculations show, indeed, an inwards decrease of the density in the BL (Hujeirat \& Camenzind 2000a; Stone \& Pringle 2000; Popham \& Sunyaev 2001).

In SSDs the pressure gradient $\nabla P_{\text {gas }}$ is neglected, whereas in ADAF the pressure adopts the profile $p \sim r^{-5 / 2}$. When comparing the gravitational to the advective time scale at the inner boundary, it is easy to find that the rate of advection increases inwards, forcing thereby the density to be minimum at the inner boundary. As a consequence, the in-flowing material at $r_{\mathrm{BL}}$ experiences a pressure-induced acceleration. Taking into account that the Coulomb coupling between the ions and electrons is $\Lambda \propto \rho^{2}$, thermal decoupling may occur, and formation of an ion-dominated torus is an inevitable phase in the evolution of accretion flows onto BHs, at least for sub-Eddington accretion.

How does heat conduction affect the structure of the ion-torus?

Generally, the temperature of both the ions and electrons increase inwards. The effect of heat conduction is to transport heat from hot into cold plasma, i.e, from inside-to-outside. In the absence of magnetic field and assuming the mean turbulent motion to be proportional to the sound speed, the innermost part of the disk will be evaporated by heat conduction of the ions, and therefore the torus starts to expand outwards (Hujeirat \& Camenzind 2000b). When large scale MFs are taken into account, the torus contracts rather than expands. The reasons are: 1) heat conduction operates parallel to the MFlines, and 2) strong MFs act to diminish the generation and dissipation of turbulence.

Unlike stars that heat up the surrounding media, BHs are heat-absorber (the surrounding coronae are not heated from below). Therefore, the ion-dominated torus contracts and may survive in the vicinity of the last stable orbit only, where MFs are pre-dominantly of large scale topology.

In addition, the location of $r_{\text {tr }}$ depends strongly on the accretion rate $\dot{\mathcal{M}}$. A large $\dot{\mathcal{M}}$ enhances the Coulomb coupling, reduces the effect of heat conduction and thereby slowing the propagation speed of the TAWs (which reduces the efficiency of the magnetic braking). Thus, the volume of the ion torus shrinks and the total power injected into the jet-plasma decreases accordingly.

\footnotetext{
5 This applies for Kerr black holes with $a<1$.
} 


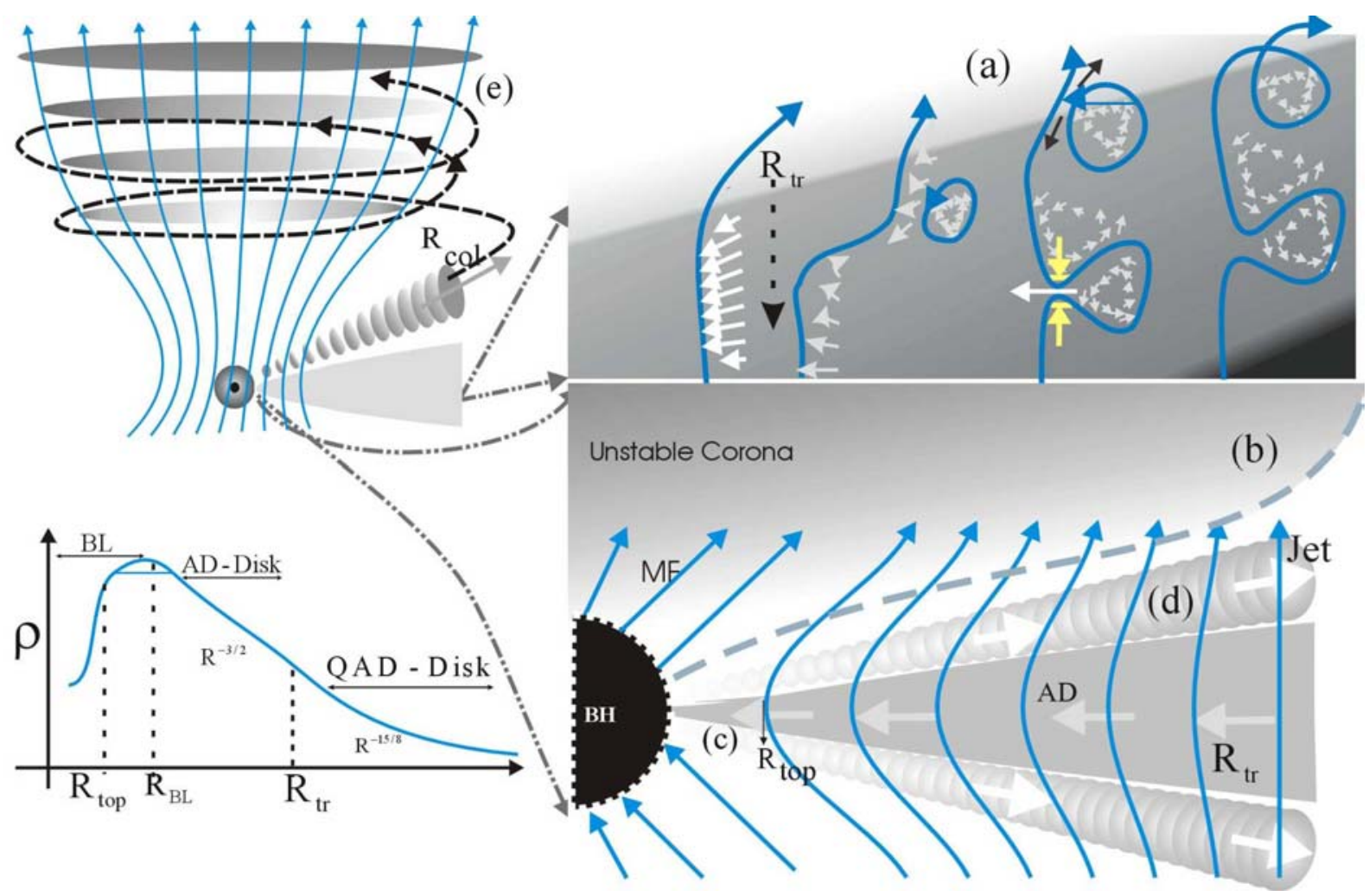

Fig. 2. A schematic illustration of the jet-disk connection. In the outer region of the disk MFs are weak and advected-around MFs by fluid motions. BH- and Parker-instability in combination with reconnection and inwards accretion amplified the MFs up to thermal equipartition. The MFs become of large scale topology and turning the disk at $r_{\mathrm{tr}}$ into advection-dominated (part $\mathbf{a}$ )). Interior to $r_{\mathrm{tr}} \mathbf{b}$ ), turbulent-generation and dissipation are suppressed, and torsional Alfvén waves become the dominant angular momentum transporter c). Torsional Alfvén waves carry angular momentum from the disk and deposit it into the thin layer above, where ions rotate super-Keplerian, virial-heated by reconnection of the TMF-lines, and start to centrifugal-accelerate outwards (part d)), The ions in the TL are associated with internal, rotational and TMF-energy to start collimation at $r_{\mathrm{col}}$, where the magnetic-diffusivity becomes vanishingly small (part e)). For clarity, a schematic description of the density profile versus radius of the model is shown.

\section{The governing equations}

When modelling flow-configurations in the vicinity of a $\mathrm{BH}$, it is necessary to use spherical geometry. Moreover, since the dynamical time scale close to the last stable orbit is extremely short, thermal decoupling between electrons and protons is possible. In this case, the set of equations to be solved consists of the continuity equation:

$\frac{\partial \rho}{\partial t}+\nabla \cdot(\rho \boldsymbol{V})=0$

the three momentum equation for the material flux:

$\frac{\partial \boldsymbol{M}}{\partial t}+\nabla \cdot\left(M \otimes V_{\mathrm{i}}\right)=-\nabla P_{\mathrm{g}}+f^{\mathrm{grav}, \text { cent,rad }}+\frac{\nabla \times B \times B}{4 \pi}+Q^{\text {visc }},(7)$

where $\rho, \boldsymbol{V}=\left(V_{\mathrm{r}}, V_{\theta}, V_{\varphi}\right), \boldsymbol{M}$ and $P$ are the density, velocity vector, material flux $\boldsymbol{M}=\rho \boldsymbol{V}$, and gas pressure $P(\doteq$ $\left.\mathcal{R}_{\text {gas }} \rho\left(T_{\mathrm{i}} / \mu_{\mathrm{i}}+T_{\mathrm{e}} / \mu_{\mathrm{e}}\right)\right)$. The subscripts "i" and "e" correspond to ions and electrons. $\mu_{\mathrm{i}}=1.23$ and $\mu_{\mathrm{e}}=1.14$. $\boldsymbol{f}^{\text {grav,cent,rad }}$ is the force vector which includes the gravitational, centrifugal and radiative forces. Quasi-Newtonian gravity is used to describe the gravity of the central BH (Paczyanski \& Wiite 1980). $Q^{\text {visc }}$ denotes the collection of second order diffusive operators.
The evolution of the MFs is followed by solving the induction equation which reads:

$\partial_{\mathrm{t}} \boldsymbol{B}=\nabla \times\left(\boldsymbol{V} \times \boldsymbol{B}-\eta_{\mathrm{mag}} \nabla \times \boldsymbol{B}\right)$.

Here, $\boldsymbol{B}=\left(B_{\mathrm{r}}, B_{\theta}, B_{\varphi}\right)=\left(B_{\mathrm{p}}, B_{\mathrm{T}}\right)$. To quench the amplification of the TMF in the TL, we adopt the magnetic turbulent diffusivity: $\eta_{\mathrm{mag}}=\alpha V_{\mathrm{AT}} H_{\mathrm{d}}$, where $V_{\mathrm{AT}}=B_{\mathrm{T}} / \sqrt{\rho}$. In solving this equation for $\boldsymbol{B}$, the solenoidal condition $\nabla \cdot \boldsymbol{B}=0$ must be satisfied everywhere and for all times (Hujeirat \& Rannacher 2001).

The dynamical time scale in the innermost region of the disk is sufficiently short, that thermal decoupling between the electrons and ions is inevitable (Rees et al. 1982; Narayan \& Yi 1995). To take this possibility into account, both equations describing the thermal evolution of the ions and electrons should be solved. In this formulation, the bulk of heat generated by turbulent dissipation goes into heating mainly the ions in virtue of their large mass compared to the electrons. The dominant cooling mechanisms for the ions are conduction and two-body interaction with the electrons. The electrons, on the other hand, are subject to various cooling processes, e.g., Bremsstrahlung, Comptonization, Synchrotron and conduction. Surprisingly, ion-conduction appears to play an important role in the innermost region of the disk, where the ions 


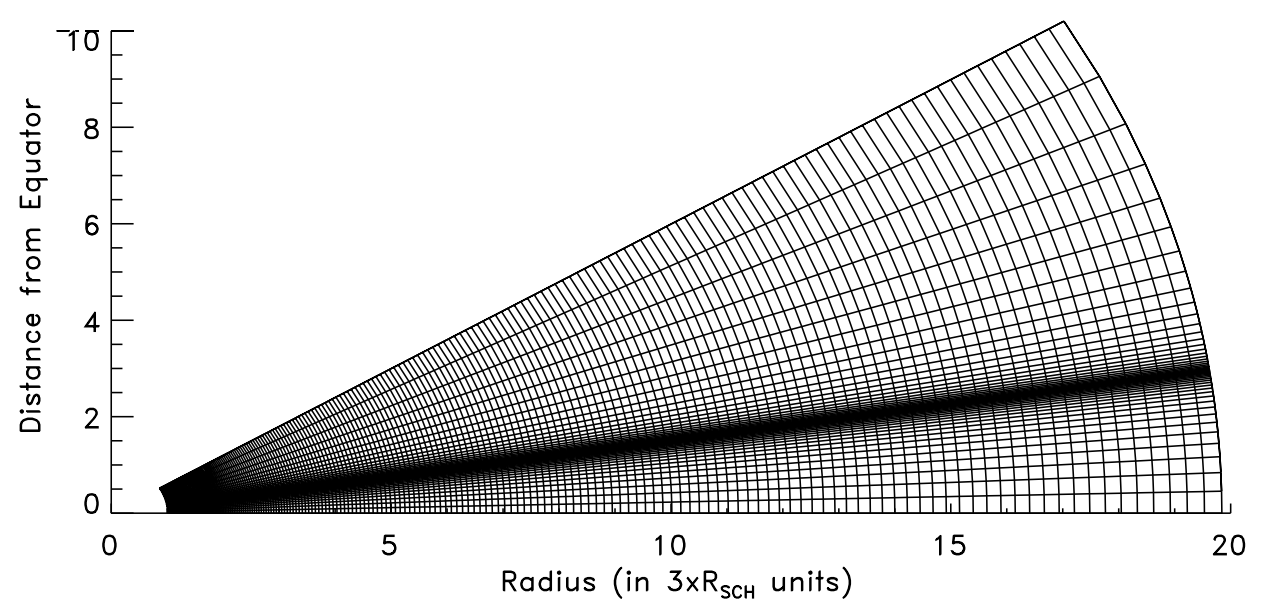

Fig. 3. A partial display of the distribution of 220 and 100 finite volume spherical cells in the radial and horizontal directions that covers the domain of calculations. Highly refined mesh distribution is adopted in the transition layer, where most of the physical variable undergo a strong spatial variation and where jet-launching occurs. The aspect ratio adopted here, i.e., the ratio of the finest volume cell to the largest one, is $\approx 10^{4}$.

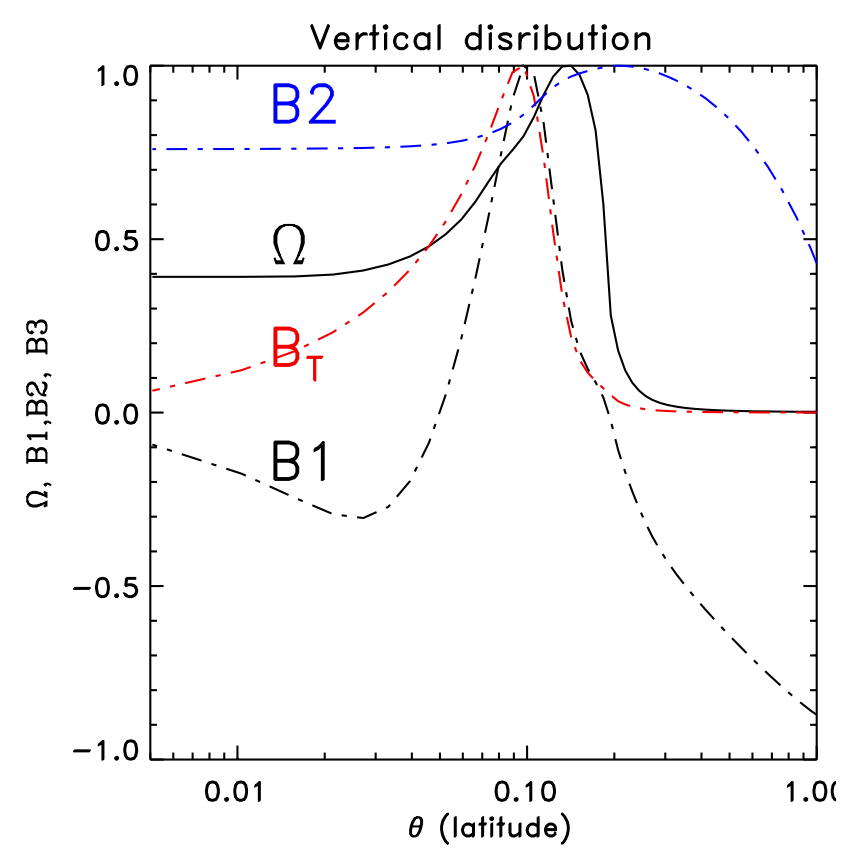

Fig. 4. The horizontal distribution of the normalized angular velocity $\Omega$, the poloidal magnetic field components $(B 1, B 2)=\left(B_{\mathrm{r}}, B_{\theta}\right)=$ $B_{\mathrm{P}}$ and the TMF $\left(B_{\mathrm{T}}\right)$ at $r=2.5$. Note the super-Keplerian rotation and the strongly enhanced strength of the MF-components in the TL.

form an ion-dominated torus and evaporate the inner part of the disk (Hujeirat \& Camenzind 2000b).

Under these conditions, the respective internal energy equations of the ions and electrons read:

$$
\frac{\partial \mathcal{E}^{\mathrm{i}}}{\partial t}+\nabla \cdot\left(\mathcal{E}^{\mathrm{i}} \boldsymbol{V}\right)=P^{\mathrm{i}}(\nabla \cdot \boldsymbol{V})+\nabla \cdot\left[\kappa_{\text {cond }}^{\mathrm{i}} \nabla T_{\mathrm{i}}\right]+\mathcal{D}-\Lambda_{\mathrm{i}-\mathrm{e}},
$$

$$
\begin{aligned}
\frac{\partial \mathcal{E}^{\mathrm{e}}}{\partial t}+\nabla \cdot\left(\mathcal{E}^{\mathrm{e}} \boldsymbol{V}\right)= & P^{\mathrm{e}}(\nabla \cdot \boldsymbol{V})+\nabla\left[\cdot \kappa_{\mathrm{cond}}^{\mathrm{e}} \nabla T_{\mathrm{e}}\right] \\
& +\Lambda_{\mathrm{i}-\mathrm{e}}-\Lambda_{\mathrm{B}}-\Lambda_{\mathrm{C}}-\Lambda_{\mathrm{syn}}
\end{aligned}
$$

where $P^{\mathrm{e}, \mathrm{i}}=\mathcal{R}_{\text {gas }} \rho\left(T_{\mathrm{i}} / \mu_{\mathrm{i}}, T_{\mathrm{e}} / \mu_{\mathrm{e}}\right), \mathcal{E}^{\mathrm{e}, \mathrm{i}}=P^{\mathrm{e}, \mathrm{i}} /(\gamma-1)$ and $\gamma=$ 5/3. D , $\Lambda_{\mathrm{B}}, \Lambda_{\mathrm{i}-\mathrm{e}}, \Lambda_{\mathrm{C}}, \Lambda_{\text {syn }}$ are the turbulent dissipation rate, Bremsstrahlung cooling, Coulomb coupling between the ions and electrons, Compton and synchrotron coolings, respectively. Here, we use the following express:

$\Lambda_{\mathrm{i}-\mathrm{e}}=5.94 \times 10^{-3} n_{\mathrm{i}} n_{\mathrm{e}} c k \frac{\left(T_{\mathrm{i}}-T_{\mathrm{e}}\right)}{T_{\mathrm{e}}^{3 / 2}}$

$\Lambda_{\mathrm{B}}=4 a c \kappa_{\mathrm{abs}} \rho\left(T^{4}-E\right)$,

$\Lambda_{\mathrm{C}}=4 \sigma n_{\mathrm{e}} c\left(\frac{k}{m_{\mathrm{e}} c^{2}}\right)\left(T_{\mathrm{e}}-T_{\mathrm{rad}}\right) E$,

where $\kappa_{\text {abs }}$ and $\sigma$ are the absorption and scattering coefficients. $n_{\mathrm{e}}, n_{\mathrm{i}}$ the electron- and ion-number densities. The radiative temperature is defined as $T_{\mathrm{rad}}=E^{1 / 4}$, and $E$ is the density of the radiative energy in the zero-moment approximation of the radiative field. For the synchrotron cooling function, the following relation is adopted (Rybicki \& Lightman 1979):

$\Lambda_{\mathrm{syn}}=\frac{E_{\mathrm{mag}}}{E} \Lambda_{\mathrm{C}}$,

where $E_{\mathrm{mag}}, E$ are the magnetic and radiative energies, respectively.

The second order operators on the RHS of Eqs. (9) and (10) are conductive operators and the conduction coefficients in cgs units read (Sandbæk \& Leer 1994):

$\kappa_{\mathrm{e}}=7.8 \times 10^{-7} T_{\mathrm{e}}^{5 / 2}, \kappa_{\mathrm{i}}=3.2 \times 10^{-8} \mathrm{~T}_{\mathrm{i}}^{5 / 2}$.

To follow the evolution of the radiative field, several assumptions have been made. Mainly, it is assumed that the field is isotropic and that the Flux-limited-Diffusion (FLD) approximation can be used to close the set of radiative moments. Thus, only the 0 -moment equation of the radiation field is solved. Since the optical depth in the disk can be large in certain regions, and small in others, FLD approximation is used to model the radiative flux appropriately in these different regions, and to 
assure the monotonicity of the flux in the transition regions inbetween. The 0 -moment of the radiation field then reads:

$\frac{\partial E}{\partial t}+\nabla \cdot(E \boldsymbol{V})=\nabla \cdot\left[\lambda_{\mathrm{FLD}} \nabla E\right]-\Lambda_{\mathrm{B}}+\Lambda_{\mathrm{C}}+\Lambda_{\mathrm{syn}}$,

where $\lambda_{\mathrm{FLD}}$ is flux limited diffusion coefficient which forces the radiative flux to adopt the correct form in optically thin and thick regions, i.e.,

$\nabla \cdot \frac{\lambda_{\mathrm{FLD}}}{\chi} \nabla E=\left\{\begin{array}{cll}\nabla \cdot \frac{1}{3 \chi} \nabla E & \text { if } & \tau \gg 1 \\ \nabla \cdot n E & \text { if } & \tau \ll 1,\end{array}\right.$

and provides a smooth matching in the transition regions. Here $\chi=\rho\left(\kappa_{\mathrm{abs}}+\sigma\right)$ and $n=\nabla E /|\nabla E|$.

\subsection{Method of solution, initial and boundary conditions}

The solution procedure is based on using the implicit finite volume solver IRMHD3 to search steady-state solution for the above-mentioned 3D axi-symmetric, two-temperature, diffusive, radiative and MHD equations in conservative form (Hujeirat \& Rannacher 2001).

We note that the strong non-linearities governing these equations may not admit steady state, but strongly timedependent or, in the best cases, quasi-stationary solutions. This implies that the terms describing the time-variation of the variables, i.e., $\partial / \partial t$, must be retained. On the other hand, since the dynamical time scale for accretion flows is shortest at the inner boundary, searching for quasi-stationary for the global flowconfiguration on these time scales could be a computationally prohibitive process.

To clarify this point, let $\partial q / \partial t=L_{\mathrm{x}} V q+\nabla P+f$ be an equation that describes the time-evolution of the vector variables $q$ under the effects of fluid motions $L_{\mathrm{x}} V q$, gradients of pressure and other forces $f$ in the interval $[a-b] . L_{\mathrm{x}}$ is a first order transport operator. We may divide $[a-b]$ into $N$ equally spaced finite volume cells: $\Delta x_{i}=(b-a) / N, i=1, N$. To follow the time-evolution of $q$ using a conditionally stable method, the time step size must fulfill the CFL-condition, which requires $\delta t$ to be smaller than the critical value: $\delta t_{\mathrm{c}}^{\mathrm{u}}=\min _{i}\left\{\Delta x_{i} /\left(V+V_{\mathrm{S}}\right)_{i}\right\}$.

If $[a-b]$ is divided into $N$ highly stretched finite volume cells, for example $\Delta x_{1}<\Delta x_{2} \ldots<\Delta x_{N}$, then the CFL-condition restrict the time step size to be smaller than $\delta t_{\mathrm{c}}^{\mathrm{nu}}=\min _{i}\left\{\left(\Delta x_{i} /\left(V+V_{\mathrm{S}}\right)_{i}\right\}\right.$, which could be much smaller than $\delta t_{\mathrm{c}}^{\mathrm{u}}$.

Therefore applying a conditionally stable method to model flows using a highly non-uniform distributed mesh has the disadvantage that the time evolution of all variables in the whole domain are artificially and severely affected by the flow behaviour on the finest cell.

Moreover, advancing the variables in time may stagnate if the flow is strongly or nearly incompressible. In this case, $V_{\mathrm{S}} \gg V$, which implies that the time step size allowed by the CFL-condition approaches zero. This indicates that conditionally stable methods are not suited for modelling such flows.

Nevertheless, one may still associate a time step size with each grid point, e.g., $\delta t_{i}^{\text {nu }}=\Delta x_{i} /\left(V+V_{\mathrm{S}}\right)$, and follow the time evolution of each variable $q_{i}$ independently. Interaction between the variables is taken into account by evaluating the spatial operators on the former time level. This method, which is called the residual smoothing method, has proved to provide quasi-stationary solutions within a reasonable computational time (Enander 1997).

Using unconditionally stable methods, i.e., fully implicit methods, the time stepping procedure is no longer restricted by the CFL-condition, and these methods potentially should be able to deal with infinite time step size. The restrictions that may arise would be due to accuracy and physical consistency considerations.

In our case, IRMHD is capable to provide accurate and physically consistent solutions when the time step size is correlated with the mesh size in the following manner:

$\delta t_{i}=\alpha \delta r_{i}$,

where $\delta t_{i}, \delta r_{i}$ are the time step size and the radial mesh increment of a finite volume cell at a given radius $r_{i}$. The coefficient $\alpha$ is a constant of order unity.

Test calculations have shown that this specially varying time stepping approach - SVTS, is most appropriate for modelling compressible flows with high Reynolds numbers on a strongly stretched mesh. If the flow is highly viscous, then SVTS can be replaced by a global uniform time step size that depends on the total time-independent residual (Turek 1994).

In the present investigation, a second order upwinding scheme has been used to treat advection. Since the temporal accuracy is at most of first order ${ }^{6}$, the solutions presented here preserve monotonicity, and therefore physically consistent.

In general, the SVTS method is likely to apply for searching quasi-steady configuration when modelling collapse of supermassive stars or cloud cores. Here, a highly stretched mesh in the radial and vertical directions is required. $\delta r_{i}$ must increase outwards strongly. On the other hand, if $\delta r_{i}$ is constant, then $\alpha$ must depend on the radius. Here, we suggest $\alpha=\alpha_{0}\left(r / r_{\text {in }}\right)$, where $\alpha$ is a constant of order unity and $r_{\text {in }}$ is the radius at the inner boundary.

The main disadvantage of the SVTS method is its inability to provide time scales for features that possess quasi-stationary behaviour that might be of astrophysical relevance. Here we suggest to use the obtained quasi-stationary solutions as initial configuration and re-start the calculations using a uniform time step size.

In Table 1 we summerize the scaling variables used in the present calculations. We have chosen the elliptical galaxy M 87 as a reference for our wind-disk model. Therefore, the central object is taken to be a super-massive non-rotating black hole. The domain of calculations is restricted to the quadrant: $D=$ $[0 \leq \theta \leq \pi / 2] \times[1 \leq r \leq 20]$. The domain $D$ is divided into $220 \times 100$ strongly stretched finite volume cells in the radial and vertical directions, respectively (Fig. 3).

\subsubsection{Boundary Conditions - BCs}

The conditions that the flow must fulfill at the boundaries are fundamentally important, especially if the solutions to

\footnotetext{
${ }^{6}$ In principle time accuracy is irrelevant if stationary solutions are sought.
} 
Table 1. The scaling variables used to reformulate the equations in non-dimensional form.

\begin{tabular}{ll}
\hline \hline Scaling variables & \\
\hline Mass & $\tilde{\mathcal{M}}=10^{8} M_{\odot}$ \\
Distance & $\tilde{R}=R_{\text {in }}=2.8 R_{\mathrm{S}}$, where $R_{\mathrm{S}}=2 G \tilde{\mathcal{M}} / c^{2}$ \\
Temperature & $\tilde{\mathcal{T}}=5 \times 10^{7} \mathrm{~K}$ \\
Density & $\tilde{\rho}=2.5 \times 10^{-12} \mathrm{~g} \mathrm{~cm}^{-3}$ \\
Velocities & $\tilde{V}=\tilde{V}_{\mathrm{S}}=\gamma \mathcal{R}_{\text {gas }} \tilde{\mathcal{T}} / \mu_{i}, \mu_{i}=1.23$ \\
Ang. velocity & $\tilde{V_{\varphi}}=V_{\mathrm{Kep}}=(G \tilde{\mathcal{M}} / \tilde{R})^{1 / 2}, \Longrightarrow \epsilon=\tilde{V}_{\mathrm{S}} / \tilde{V}_{\varphi}$ \\
Magnetic fields & $\tilde{B}=\tilde{V}_{\mathrm{S}} / \sqrt{4 \pi \tilde{\rho}}$ \\
\hline
\end{tabular}

be sought are quasi-stationary and contain inflow-outflow components.

In the present study, the following boundary conditions have been used:

- Through the outer boundary, $r_{\text {out }}$, and within the interval ${ }^{7}$ $\left[0 \leq \theta \leq \theta_{\mathrm{d}}\right]$, the constant accretion rate $\dot{\mathcal{M}}=0.01 \times \dot{\mathcal{M}}_{\text {Edd }}$ has been assumed. The values of the variables, such as density, temperature, radial and angular velocities are taken from standard thin disk theory (Frank et al. 1991). In terms of Table $1, \rho\left(r_{\text {out }}, \theta\right)=\mathrm{e}^{-\left(\theta_{\mathrm{d}} / \theta\right)^{2}}$ and $T\left(r_{\text {out }}, \theta\right)=1$. Moreover, we set $V_{\text {theta }}=0$ and $T_{i}=T_{\mathrm{e}}$. The poloidal magnetic field is taken to be in equipartition with thermal energy of the plasma.

- In the interval $\left[\theta_{\mathrm{d}} \leq \theta \leq \pi / 2\right]$ and along $r_{\text {out }}$, we set $\partial U / \partial r=V=\Omega=0$. A vanishingly small turbulent magnetic diffusivity, $v_{\mathrm{mag}}$, is assumed in this interval. This is essential in order to avoid artificial interaction between the variables in ghost zones ${ }^{8}$ with outflows that possibly form within the domain of calculations. In terms of Table 1 , we use $\rho=10^{-4}$ and $T=10^{3}$. We specify $B_{\theta}$ in the ghost zones, whereas the $B_{\mathrm{r}}$ is computed by solving the $r$-component of Eq. (8). This is a useful strategy essential in order to avoid space-freezing of the MFs to the grid points at the outer boundary.

- At the inner boundary, $r_{\text {in }}$, and within $[0 \leq \theta \leq \pi / 2]$, we impose stress-free boundary conditions, i.e., all operators containing $\partial / \partial r$ must vanish across $r_{\text {in }}$. This is an essential requirement in order to avoid upward-propagation from down-stream regions. For the radial momentum equation which require the radial velocity to be known at the $r_{\text {in }}$, we use an extrapolation procedure. Thus, if the velocity at the interface $j-1$ is negative (see Fig. 6), extrapolation is employed. If the it is positive, we set $U\left(r_{\mathrm{in}}, \theta_{\mathrm{k}}\right)=0$, so to avoid material flux into the domain of calculations from down-stream regions, i.e., from the $\mathrm{BH}$. The vertical velocity is assumed to vanish at $r_{\text {in }}$. The equations describing the evolution of the density and temperature contain first order transport operators only. Therefore they accept upwind BCs

${ }^{7} \theta_{\mathrm{d}}=\tan ^{-1}\left(H_{\mathrm{d}} / r\right) \approx 0.1$. Note that we use spherical geometry in which $\theta$ increases upwards from zero at the equator to $\pi / 2$ at the axis of rotation.

8 The ghost zones are located exterior to the outer boundary, and their main effect is, if necessary, to supply virial hot inflows with very low density. that are specified at the outer boundary. It is therefore mathematically inconsistent to specify additional conditions on the down stream boundary.

- Special attention was given to conditions that MFs must fulfill at the inner and outer boundaries. The electromotive force, $\varepsilon_{j, k}$, is defined on the cell corners (Fig. 6), and consists of two contributions:

$$
\varepsilon_{j, k}=[\boldsymbol{V} \times \boldsymbol{B}]_{1}+\left[\frac{\eta_{\mathrm{mag}}}{r}\left(\frac{\partial}{\partial \theta} B_{\mathrm{r}}-\frac{\partial}{\partial r} r B_{\theta}\right)\right]_{2}
$$

The first contribution, []$_{1}$, corresponds to fluid motionrelated effects, whereas the second contribution contains magnetic diffusivity effects. Thus, In order to avoid upwards propagation of the diffusivity-induced informations, we set $\partial\left(r B_{\theta}\right) / \partial r=0$ at $r_{\text {in }}$ and at $r_{\text {out }}$.

- Along the equator and on the axis of rotation, the radial velocity, angular velocity, density, e/p-temperatures and the $\theta$-component of the PMF $\left(B_{\theta}\right)$ are set to acquire symmetric conditions, i.e, $\partial / \partial \theta=0$, whereas the vertical velocity, the $r$-component of the PMF $\left(B_{\mathrm{r}}\right)$, and the TMF $\left(B_{\mathrm{T}}\right)$ fulfill asymmetric BCs.

\subsubsection{Initial Conditions - ICs}

We first construct a geometrically thin Keplerian disk that match the conditions imposed at the outer boundary. Therefore, the starting profiles of the density, temperature, radial and angular velocities are similar to those in standard thin accretion disks ${ }^{9}$. Thus, the disk-plasma is taken to be isothermal and in hydrostatic equilibrium in the vertical direction. This yields the exponential decrease of the density $\rho(r, \theta)=\mathrm{e}^{-\left(\theta / \theta_{\mathrm{d}}\right)^{2}}$. Moreover, the starting configuration has $V=0, T_{\mathrm{e}}=T_{i}$ and $B_{\mathrm{T}}=0$. A poloidal magnetic field of large scale topology has been used as IC. Its intensity has been computed, so to be in equipartition with thermal energy of the disk-plasma at the equator.

Above the disk, a hot and tenuous corona ( $T=T_{\text {virial }}$, and density $\rho(t=0, r, \theta)=10^{-4} \rho(t=0, r, \theta=0)$ is set to sandwich the disk from both sides.

\footnotetext{
9 The angular velocity is modified so to match the Paczynski-Wiita gravitational potential (Hujeirat et al. 2002).
} 


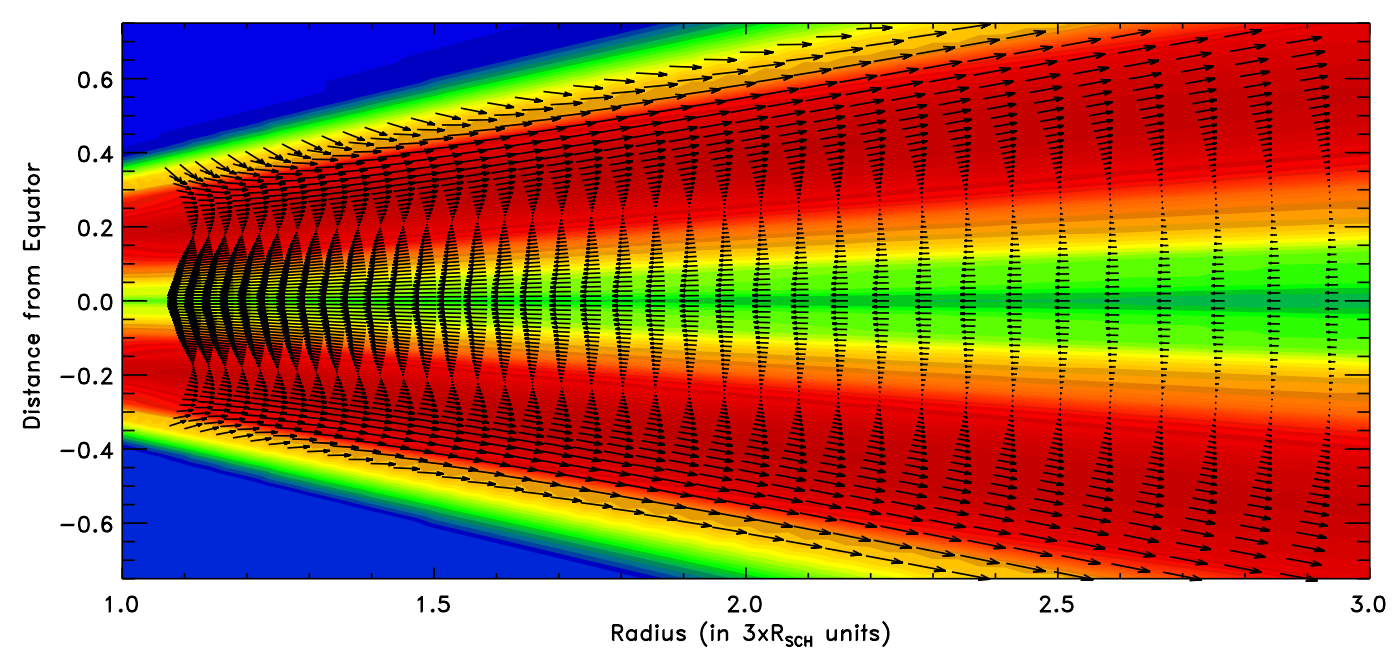

Fig. 5. The distribution of the velocity field in the innermost part of the disk, superposed on the logarithmic-scaled ratio of the ion- to electrontemperatures (red color corresponds to high ratios and blue to low-ratios). (This figure is available in colour in electronic form.)

\section{Results: Formation of the super-Keplerian layer}

When an isolated Keplerian-rotating particle in the equatorial near-plane is shifted to higher latitudes while conserving its angular momentum, its rotation becomes super-Keplerian locally, and therefore it starts to move outwards. Moreover, noting that $\tau_{\text {TAW }}$ decreases strongly inwards, a transition layer between the disk and the hot tenuous corona starts to form from inside-to-outside, where the matter rotates super-Keplerian (Fig. 2). Based on a previous calculations (Hujeirat et al. 2002b), it was found that: 1) the angular velocity in the TL adopts the approximate power law profile $\Omega \sim r^{-5 / 4}, 2$ ) ions cool predominantly through fast outflows, and 3) the amplification of the generated TMF is equilibrated through outward advection and magnetic-quenching (i.e., we artificially increased the coefficient of the magnetic diffusion in the TL to allow a flux-loss of the TMF). In the TL, one possible solution for the radiative MHD equations is a self-similar solution in which the variables obey the following power-laws:

$\Omega \sim r^{-5 / 4}, \rho \sim r^{-3 / 2}, T_{\mathrm{i}} \sim r^{-1 / 2}, U_{\mathrm{r}} \sim r^{-1 / 4}$,

$B_{\mathrm{P}} / B_{\mathrm{T}} \sim$ const. $=\epsilon$,

where $\epsilon=H_{\mathrm{W}} / r$, and $H_{\mathrm{W}}$ is the width of the TL. The angular velocity here decreases with radius much slower than the disk, hence the plasma is centrifugally accelerated outwards (see Fig. 7). The above-correlation between $B_{\mathrm{p}}$ and $B_{\mathrm{T}}$ in the TL results from the fact that the rates of advection and generation of the TMF are equal, i.e, $\tau_{\text {gen }}=\tau_{\text {adv }}$. In terms of Eq. (8), we obtain $B_{\mathrm{p}} / B_{\mathrm{T}}=H_{\mathrm{W}} / r=\epsilon$.

The most prominent properties of this solution are:

1. The ratio of heating to advection time scale of the ions is of order unity, i.e., $\tau_{\mathrm{h}} / \tau_{\mathrm{adv}} \approx 1$. The ratio of heating to Coulomb-cooling time scale of the ions is: $\tau_{\mathrm{h}} / \tau_{\text {Coul }} \sim$ $r^{-1 / 2}$. This implies that thermal coupling between electrons and ions diverges with distance from the origin, and gives rise to multi-component jet-plasmas (Fig. 5). Furthermore, ions cool pre-dominantly through advection, i.e., adiabatic expansion.

2. The ratio of the amplification of the TMF to the flux-loss time scale is of order unity. The same applies to the ratio of the advection to amplification time scale. Thus, besides heating the plasma via magnetic reconnection of the the TMF-lines (see for example Fig. 8 for the case of weak magnetic fields), this implies that a significant fraction of the toroidal magnetic flux is advected outwards, and which thereafter acts to collimate the flow. On the other hand, the magnetic diffusivity in the TL scales: $\eta_{\text {mag }} \approx$ $H_{\mathrm{W}}\left(B_{\mathrm{p}} / B_{\mathrm{T}}\right) V_{\varphi} \sim \epsilon r^{3 / 4}$. This $\eta_{\mathrm{mag}}$-description must break down at a certain radius $r_{\text {col }}$, where $\eta_{\text {mag }}$ becomes negligibly small and collimation starts to be efficient.

3. The rate of the outward-oriented material flux in the TL reads:

$$
\dot{\mathcal{M}}_{\mathrm{W}}(r)=\dot{\mathcal{M}}_{\mathrm{W}}\left(r=r_{\text {in }}\right)+\epsilon^{2} \dot{\mathcal{M}}_{\mathrm{d}}\left(r=r_{\text {in }}\right)\left(\left(\frac{r}{r_{\text {in }}}\right)^{1 / 4}-1\right) \text {, }
$$

where $\dot{\mathcal{M}}_{\mathrm{W}}\left(r=r_{\text {in }}\right)$ and $\dot{\mathcal{M}}_{\mathrm{d}}\left(r=r_{\text {in }}\right)$ are the wind-flux rate and the disk accretion rate at the inner boundary. In writing Eq. (16) we have r-integrated the continuity equation: $\partial \dot{\mathcal{M}}_{\mathrm{W}} / \partial r=\left.H_{\mathrm{d}}(\rho V)\right|_{\theta_{\mathrm{d}}}=\epsilon^{2} r^{-3 / 4}$, using $H_{\mathrm{d}}=r \sin \theta_{\mathrm{d}}$, $\epsilon=H_{\mathrm{d}} / r$ and $V=\left(H_{\mathrm{d}} / r\right) U . \theta_{\mathrm{d}}$ corresponds to the latitudinal angle between the equator and the surface of the disk is spherical geometry.

Thus, the wind flux increases with radius, although it is a rather weak dependence.

4. the associated angular momentum flux with the wind increases linearly with distance from the central $\mathrm{BH}$ :

$\dot{\mathcal{J}}=$ const. $r$

which applies for $r \leq r_{\mathrm{tr}} \approx 10-20 R_{\mathrm{LSO}}$.

The global energy distribution in the disk and jet region can be well represented by the Bernoulli number $(\mathrm{Be})$. Flows with total positive energy are gravitationally unbound, and potentially 
they may propagate to infinity, whereas flows with negative total energy are gravitationally bound and they end their motion inside the BH. However, if the flow is dissipative, energy exchange between different parts of the flow may occur, so that an inflow-outflow configuration is possible. Figure 10, for example shows that $\mathrm{Be}$ is everywhere negative safe the TL, where it attains large positive values.

We note that the outflow is sufficiently strong to shift the poloidal magnetic field (PMF) lines outwards, whereas the large magnetic diffusivity prevents the formation of large electric currents along the equator. In the case of weak MFs ( $\beta \leq 0.1$, see Fig. 8), our calculations reveal rather weak outflows, which is reasonable, since a low $\beta$ yields a longer $\tau_{\text {TAW }}$, which may become longer than the dynamical time scale. Moreover, low $\beta$ s inflows force MFs to establish a monopole like-configuration (i.e, a one-dimensional MF-topology which is not appropriate for producing strong TMFs). In this case, the rate of TMF-generation is considerably reduced. TAWs become inefficient at magnetic-braking the disk, reducing therefore the power required for launching jets. This indicates that weakly magnetized disks are in-appropriate for launching powerful jets, and that magnetized accretion flows are more suited for jet-production than their HD-counterparts. Comparing the flux of matter in the wind region to that in the disk, it has been found that $\dot{\mathcal{M}}_{W} / \dot{\mathcal{M}}_{\mathrm{d}}=$ const. $\sim \epsilon^{2}$. The angular momentum flux associated with the wind is $\dot{\mathcal{J}}_{\mathrm{W}} / \dot{\mathcal{J}}_{\mathrm{d}}=a\left(\dot{\mathcal{M}}_{\mathrm{W}} / \dot{\mathcal{M}}_{\mathrm{d}}\right) r^{1 / 4}$, where " $a$ " is a constant of order unity. Consequently, at 500 gravitational radii almost $15 \%$ of the total accreted angular momentum in the disk re-appears in the wind. To clarify why the TL is geometrically thin, we note that the ratio:

$\frac{\tau_{\mathrm{TAW}}}{\tau_{\mathrm{adv}}} \sim\left(\frac{r}{H_{\mathrm{d}}}\right)\left(\frac{V_{\mathrm{A}}}{U}\right) \sim \frac{r}{H_{\mathrm{d}}}>1$,

where $\tau_{\text {adv }}$ is the advection time scale. The last inequality implies that angular momentum in the TL will be advected outwards more efficiently than being extracted through TAWs to higher latitudes. Furthermore, since the flow in the TL is highly dissipative, TMF-lines tend to close in the TL, thereby considerably reducing the efficiency of angular momentum transport to much higher latitudes. Also, as the flow in the TL rotate super-Keplerian, the normal component of the centrifugal force tend to shift the matter towards the equator.

On the other hand, the corona above the TL has been found to be dynamically unstable. Unlike normal stars that heat up the surrounding corona from below, in the absence of other energy sources, black holes cannot supply the surrounding corona with heat, hence they start to collapse dynamically.

To elaborate this point, let us compare the conduction time scale with the dynamical time scale along $\mathrm{B}_{\mathrm{P}}$-field at the last stable orbit of a SMBH:

$\frac{\tau_{\text {cond }}}{\tau_{\text {dyn }}}=\frac{r \rho U_{r}}{\kappa_{0} T_{\mathrm{i}}^{5 / 2}}=4.78 \times 10^{-4} \rho_{10} T_{\mathrm{i}, 10}^{-5 / 2} \mathcal{M}_{8}$,

where $\rho_{10}, \mathrm{~T}_{\mathrm{i}, 10}$ and $\mathcal{M}_{8}$ are respectively in $10^{-10} \mathrm{~g} \mathrm{~cm}^{-3}$, $10^{10} \mathrm{~K}$ and in $10^{8} \mathcal{M}_{\odot}$ units. This is much less than unity for most reasonable values of density and temperature typical for AGN-environments. In writing the above equation we have optimistically taken the upper limit $c / \sqrt{3}$ for the velocity, and

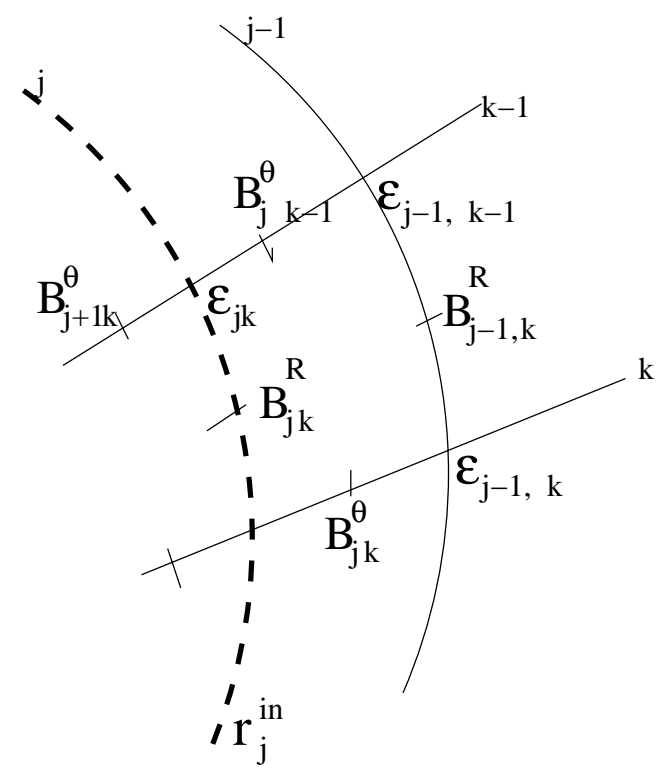

Fig. 6. The location of the poloidal magnetic field components and the electromotive forces in a finite volume cell $(j, k)$ at the inner boundary.

set $\kappa_{0}=3.2 \times 10^{-8}$ for the ion-conduction coefficient. When modifying the conduction operator to respect causality, we obtain $\tau_{\text {cond }} / \tau_{\text {dyn }} \leq U_{\mathrm{r}} / c$, which is again smaller than unity.

This agrees with our numerical calculations which rule out the possibility of outflows from the corona, and in particular not from the highly unstable polar region of the $\mathrm{BH}$, where matter is neither magnetic- nor centrifugal-supported against the central gravity of the $\mathrm{BH}$.

\section{Formation of electron-proton jets: Summary}

In this paper we have presented a model for initiating jets from systems containing BHs surrounded by accretion disks. The model is based on the following sub-structures:

1. A weakly magnetized accretion disk in the outer region. Balbus-Hawley instability amplifies the weak MFs up to thermal equipartition which, in combination with the Parker instability and inward-advection of matter, re-generate a PMF of large scale topology. This large scale PMF turns the disk at $r_{\text {tr }}$ into advection-dominated. However, since this occurs on the dynamical time scale which is radius-dependent, $r_{\text {tr }}$ is relatively large, or alternatively is close to the black hole. The former possibility is observationally inconsistent, as this would force the disk to truncate at large radii and terminate accretion. The latter possibility is plausible: assume that the flow at $r_{\text {tr }}$ is freely falling, and has the vertical width of $H_{\mathrm{d}} / r=1 / 30$. Using $\rho(r, \theta=0)=\rho\left(r_{0}, \theta=0\right)\left(r_{\mathrm{BL}} / r\right)^{3 / 2}$ and $B_{\mathrm{p}}(r, \theta=0)=$ $B_{\mathrm{p}}\left(r_{0}, \theta=0\right)\left(r_{\mathrm{BL}} / r\right)^{2}$, one can easily verify that MFs would terminate accretion if $r_{\mathrm{tr}} \geq 16 r_{\mathrm{LSO}}$, where $r_{\mathrm{LSO}}$ is the last stable radius. Here we have assumed that whatever is the strength of the MF, the magnetized inflow interior to $r_{\mathrm{LSO}}$ is gravitationally bound and will be accreted, together with the MF, into the hole. In this region, we anticipate large scale MFs to change into a monopole like-configuration. 


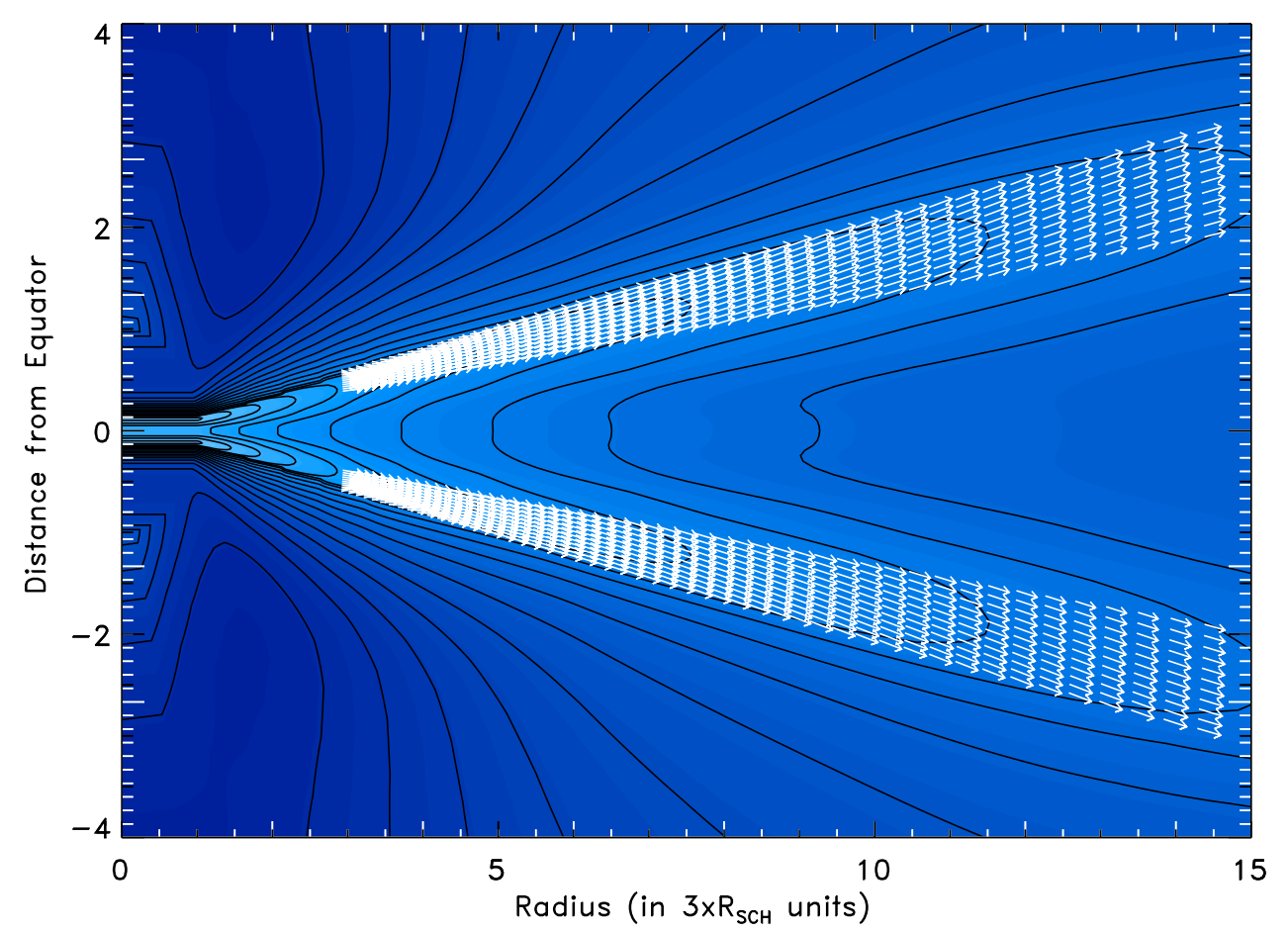

Fig. 7. The distribution of the velocity field in the transition region superposed on equally-spaced isolines of the angular velocity $\Omega$. The strong-decrease of $\Omega$ with radius in the equatorial region relative to its slow-decrease in the TL is obvious.

Thus, an advective disk threaded by large scale MFs must be located inside $10-20 r_{\mathrm{LSO}}$, depending on the accretion rate.

2. The plasma in the innermost region of the disk is turbulentfree. Under such circumstances, the rate of inflow depends strongly on the efficiency ofthe TAWs at extracting angular momentum from the disk and feeding the plasma in the TL. The time scale at which the TL forms is essentially the TAW crossing time $\tau_{\mathrm{TAW}}=H_{\mathrm{W}} / V_{\mathrm{A}}^{\mathrm{P}}$, which is approximately equal to, or shorten than the dynamical time scale. Noting that $\tau_{\text {TAW }}$ is shortest at the smallest radius, we may conclude that the TL forms from inside-to-outside. The latter process is essential for explaining the deep penetration of the accreted plasmas in the gravitational well of the central BHs, and the origin of their large kinetic energies when they re-appear in the form of jets.

3. In the region $r_{\text {top }} \leq r \leq r_{\text {tr }}$, TAWs are the dominant angular momentum carrier. The transport proceeds vertically and on approximately the dynamical time scale, which requires the disk, again, to be advection-dominated for stably supplying the jet with angular momentum. The angular momentum associated with TAWs is deposited in the turbulent-diffusive TL between the disk and the corona, where a significant of the shear-generated TMFs reconnect and heat the plasma up to the virial temperature. The strong magnetic fields in the TL force the electrons to cool rapidly. Taking into account the fast outwards-oriented motion of the flow, the outflow is essentially a two-temperature iondominated plasma. The TMFs here are in thermal equipartition with the ions, whereas the poloidal component is in equipartition with the electrons. Such a flow-configuration is essential for explaining the origin of the excessive radio luminosities observed in several AGNs.

Besides the fact that the large magnetic-diffusivity in the TL lengthens the time scale of TAWs to cross the TL compared to the local dynamical time scale (which is necessary for maintaining a geometrical thin TL), reconnection of the TMF-lines terminates magnetic braking and yields a trapping of the angular momentum. Consequently, the virialheated ions are then forced to rotate super-Keplerian and centrifugal-accelerate outwards.

Another argument in favor of the thin geometrical structure of the TL is that the corona is thermally unstable. The reasons are: 1) absence of heating from below, 2) centrifugal forces are weak, and 3) Lorentz forces exerted on the plasma in the polar region of the $\mathrm{BH}$ are negligibly small.

4. The plasma in the TL is tenuous, highly advective, twotemperature and ion-dominated, which gives rise to the formation of electron-proton jets. The shear-generated TMFs in the TL are in thermal equipartition with the ions and in super-equipartition with the thermal energy of the electrons. Such a strong TMF in the TL is necessary for explaining the observed excess of radio luminosities such as in M 87 (see Fig. 11) and GRO 1655-40.

We note that if the axi-symmetric assumption is relaxed, there might be still ways to transport angular momentum from the disk into the corona without forming a dissipative TL. Such ways can be explored using a full 3D implicit radiative MHD solver, taking into account Bremsstrahlung and Synchrotron coolings, heat conduction, and able to deal with highly stretched mesh distributions. Such solvers are not available to date, and therefore beyond the scope of the 


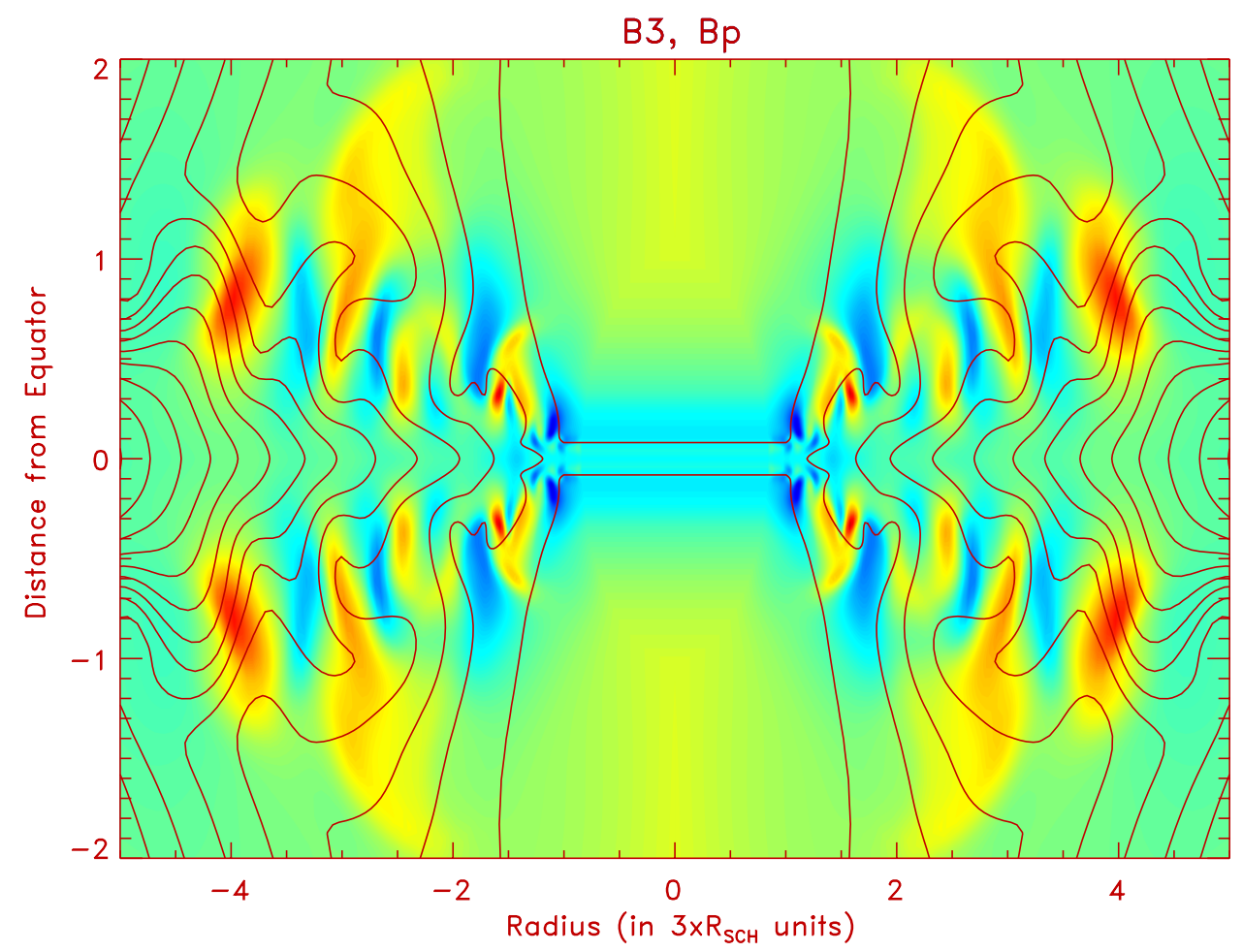

Fig. 8. The distribution of 30 weak poloidal magnetic field lines around a non-rotating BH after 10 orbital periods at the inner boundary (black lines). The TMF-distribution is shown as well (blue color corresponds to large positive values, and red to negative values). In this run we set $\beta=1 / 20$, the disk is relatively hot $\left(T\left(\theta=0, r=r_{\text {out }}=10^{-1} T_{\text {virial }}\right)\right.$ and thin $\left(\dot{\mathcal{M}}=10^{-3} \dot{\mathcal{M}}_{\text {Edd }}\right)$. Obviously, the poloidal and toroidal MF components are strongly time-dependent and attain maximum values in the TL (to be compared with Fig. 4 in Hujeirat et al. 2002b where an initially large $\beta$-inflow is assumed). (This figure is available in colour in electronic form.)

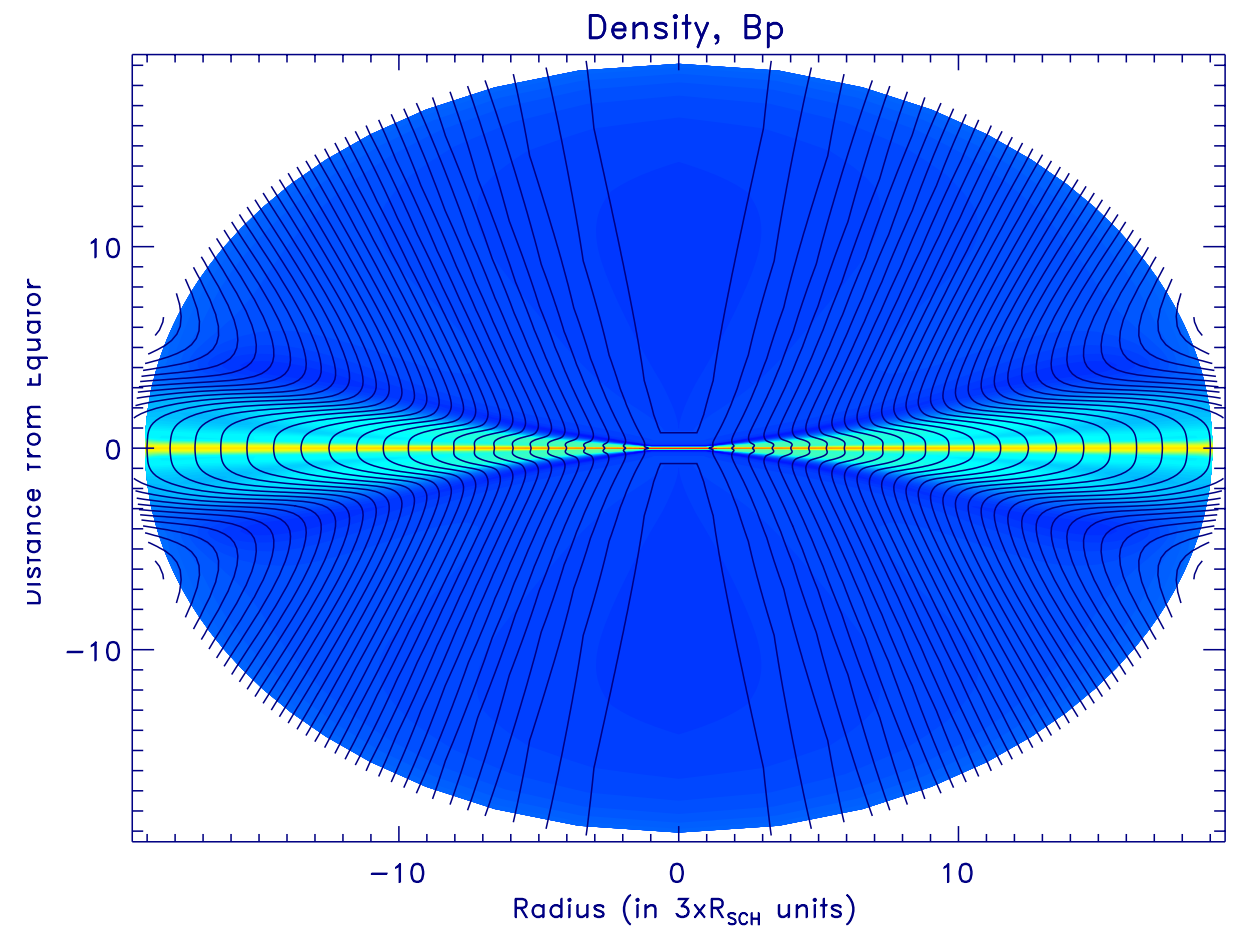

Fig. 9. 30 equally-spaced isolines of the poloidal-component $B_{\mathrm{P}}$ (black lines) and the density-distribution (yellow color corresponds to very high density-values, blue to middle and violet to low values). (This figure is available in colour in electronic form.) 


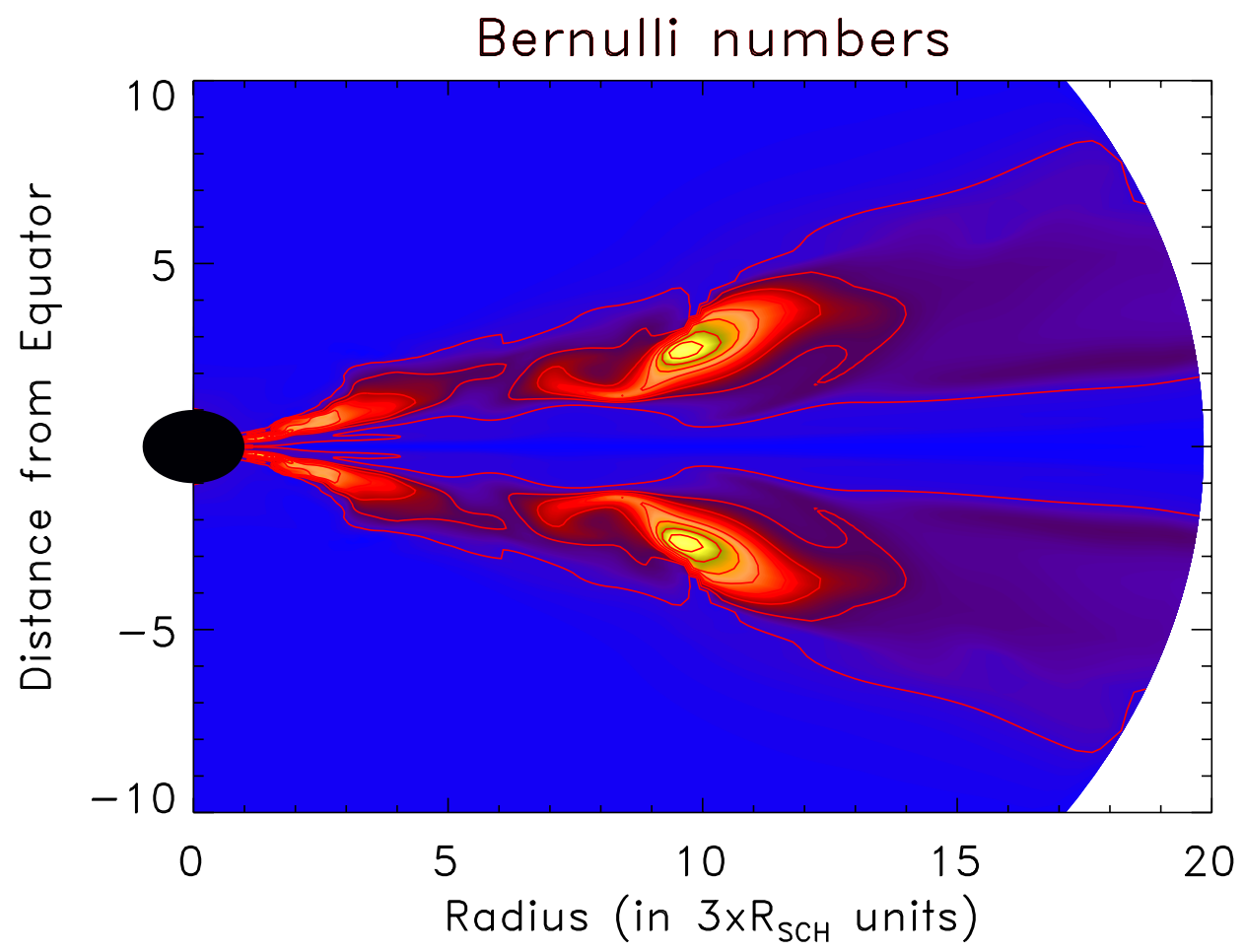

Fig. 10. A snap-shot of the distribution of the Bernoulli number in two-dimensional quasi-stationary calculations. The decrease from large to low positive values is represented via yellow, green and red colors. The blue color corresponds to negative values. The figure shows two ejected gravitationally unbound blobs of large positive energies in the TL (This figure is available in colour in electronic form.)

present paper. On the other hand, since the region of interest is located in the vicinity of an axi-symmetric black hole, the axi-symmetric assumption might be an appropriate description for the flow in the TL and in the disk. Furthermore, in the absence of heating from below, coronae around black holes have been found to be dynamically unstable to heat conduction (Hujeirat et al. 2002). In order to hold a BHcorona against dynamical collapse, the MFs threading the corona must be sufficiently strong. These, in turn, force the gyrating electrons to cool rapidly through emitting synchrotron radiation. In the absence of efficient heating mechanisms for the plasma in the corona, this cooling may even runaway.

Consequently, coronae around BHs most likely will diminish, and the robust way to form jets would be through the formation of TLs, which might be a natural consequence of accretion flows onto BHs. In this case, using IRMHD, or similarly, a 3D axi-symmetric radiative MHD solver, taking into account the relevant physical process and using adaptive mesh refinement are likely to capture the inflowoutflow configuration accurately.

It should be noted here that test calculations have shown that the formation of the TL is strongly sensitive to the spatial resolution used. This results from the fact the the TL evolves from inside-to-outside. If the spatial treatment of the transport operators in the vicinity of the inner radius is not sufficiently accurate, the TAWs may fail to transport the required amount of angular momentum to build up an interface with zero effective gravity. Furthermore, the TL may blow up thermally if the diffusive operators dominate the transport operators at the interface between the TL and the underlying disk.

5. While a part of the generated TMF is advected outwards with the centrifugally accelerated plasma in the TL, a significant fraction will still undergo reconnection and heats the heavy particles up to the virial temperature. To clarify this point, we may compare the rate of heating via magnetic dissipation with the generation rate of the TMF:

$$
\frac{\left(1 / \tau_{\text {Diss }}\right)}{\left(1 / \tau_{\text {gen }}\right)}=\frac{\tau_{\text {gen }}}{\tau_{\text {Diss }}} \approx \epsilon\left(\frac{B_{\mathrm{T}}}{B_{\mathrm{p}}}\right)\left(\frac{\eta_{\mathrm{mag}}}{H_{\mathrm{W}} V_{\varphi}}\right)=O(1),
$$

where we have used $\eta_{\text {mag }}=H_{\mathrm{W}} V_{\mathrm{A}}^{\mathrm{T}}$ and $B_{\mathrm{p}} / B_{\mathrm{T}}=H_{\mathrm{W}} / r=\epsilon$. $V_{\mathrm{S}}$ is the sound speed of the virial-hot ions, which is approximately equal to $V_{\mathrm{A}}^{\mathrm{T}}$ and $V_{\varphi}$.

Consequently, almost $50 \%$ of the generated toroidal magnetic energy is dissipated in the TL, while the rest is advected outwards.

6. In the present paper, we have assumed that reconnection heats both the electrons and the heavy particles, equally. This is a reasonable assumption, as dissipation of MHD waves may inject heat directly into the electrons (Quataert 1998), whereas turbulent dissipation preferentially heats the heavy particles (Rees et al. 1982). If synchrotron emission is not efficient, then collective plasma waves have been found to enhance the electron-proton interactions, and a single temperature for both plasma components would result (Begelman \& Chiueh 1988). Nevertheless, a detail modelling of magnetic reconnection would require 


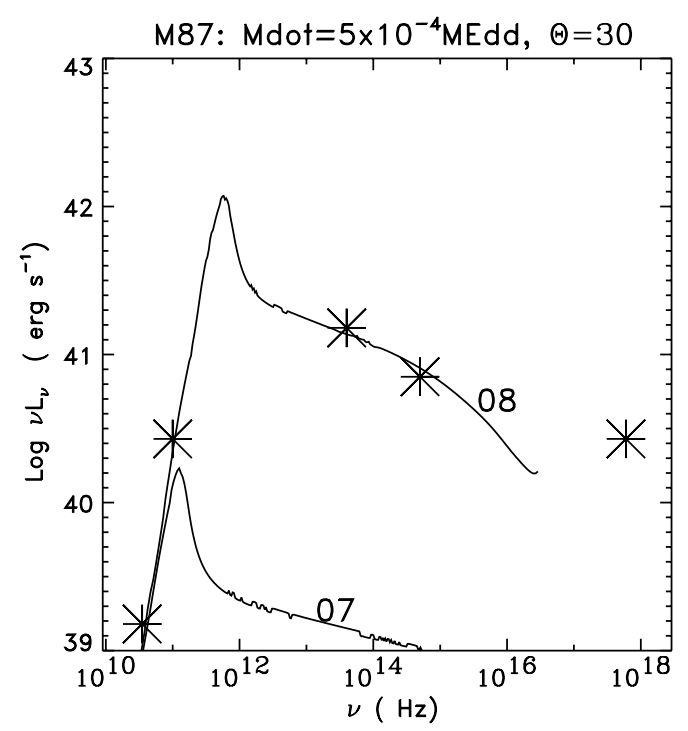

Fig. 11. The SED of the disk-jet model of the elliptical galaxy M 87 (for further details see Biretta et al. 2002; Di Matteo et al. 2003). In the present calculations, an accretion rate of $5 \times 10^{-4} \dot{\mathcal{M}}_{\text {Eddington }}$ and $T=5 \times 10^{6} \mathrm{~K}$ is set to enter the domain of calculations through the outer boundary, which is located at 150 Schwarzschild radii from the central SMBH. The vertical scale height of the disk at the outer boundary is taken to be $H \approx 0.1 R_{\text {out }}$. Additionally, a hot tenuous corona is set to sandwich the optically thin disk. The calculated profiles (solid lines) are superposed on the observational data (asterisks, see Fig. 3 in Di Matteo et al. 2003). The line 07 corresponds to a model with zero TMF (i.e., TMF $=0$ ), but a PMF which is set to be in equipartition with the thermal energy of the electrons. The line 08 is similar to 07 , but the TMF is allowed to develop and reach values beyond equipartition with respect to the thermal energy of the electrons in the TL. The above SED has been obtained by solving the radiative transfer equation in 4-dimensions, taking into account the Kompaneets operator for consistently modelling Comptonization (see Hujeirat \& Camenzind 2002a; Hujeirat 2003, for further details). 400 non-linearly distributed frequency points have been used to cover the frequency space, and $125 \times 40$ finite volume cells to cover the domain $D$ (see Sect. 4.1).

incorporating of complicated physical processes that operate on micro-scales (see Lazarian \& Vishniac 2000), which is beyond the scope of the present study.

7. Since the pressure-gradient vanishes at the horizon, a rarefaction wave starts to move from inside-to-outside, leaving the matter behind its front free-falling. The resulting density profile has a global maximum at $r_{\mathrm{BL}}\left(\ll r_{\mathrm{tr}}\right)$, and a minimum at the inner boundary $r_{\text {in }}$, where the dynamical time scale is shortest, and where the strength of Coulomb coupling between the ions and electrons is weakest. Consequently, the disk truncates at $r_{\mathrm{BL}}$ and an highly advective ion-dominated torus emerges in the BL. The MF in the ion-torus change topologically from large scale into monopole. Such a change is associated with a significant loss of magnetic flux in the hole, which is necessary for preventing magnetic termination of accretion.

8. When does the outflow start to collimate into jet? A significant part of the shear-generated TMFs reconnect in the TL and heat the plasma. The other part is advected outwards with the plasma on the dynamical time scale. Inspection of Eq. (1) shows that the ratio of the viscous to the advective time scale of angular momentum in the TL reads:

$\frac{\tau_{\mathrm{visc}}}{\tau_{\mathrm{adv}}} \approx \frac{r V_{\mathrm{A}}}{v_{\mathrm{tur}}}$.

In writing this approximation we have inserted $V_{\mathrm{A}}^{\mathrm{T}}=$ $B_{\mathrm{T}} / \sqrt{\rho}=V_{\varphi}$, which is applicable for the plasma in the TL. Therefore, given the critical radius $r_{\text {col }}\left(\doteq v_{\text {tur }} / V_{\mathrm{A}}\right)$ at which both viscous and advective time scale are equal, collimation will start for $r \geq r_{\mathrm{col}}$. This is easy to fulfill, as $V_{\mathrm{A}}$ increases with distance from the $\mathrm{BH}$, or remain constant, whereas $v_{\text {tur }}$ must decrease. This gives rise to extremely large ratio of $\tau_{\text {visc }} / \tau_{\text {adv }}$. Therefore, beyond $r_{\text {col }}$, particle-trajectories of motion are dictated by MFs.

Nevertheless, the process of collimation requires investigating the magnetic and centrifugal forces exerted on the plasma particles. Based on previous studies (see Fendt \& Memola 2001, and the references therein), $r_{\text {col }}$ is most probably located close to the light cylinder. The associated toroidal magnetic component in combination with the PMF-component will force the plasma to collimate most likely through the pinching effect.

Finally, similar to the Blandford \& Znajek (1977) scenario, the vertical transport of angular momentum via TAWs in our model is mediated almost without advection of matter. This gives rise to high collimated and extremely low mass-loaded jets. The global jet-disk configuration in our model pronounces the role of the central engine that may play in powering jets (Koide et al. 2002). A spinning BH is likely to feed the jet with extra rotational energy through the frame dragging effect, whereas a non-rotating hole may have an opposite effect: it extracts energy from the plasma in the TL, reducing thereby the energy budget of the outflow, hence yielding a less powerful jet.

The outflows presented in this paper serve as seeds for jets rather than for winds. We note that the mechanisms underlying the formation of these outflows operate efficiently in the vicinity of the central black hole. They fail to operate in regions that are beyond 10-20 Schwarzschild radii from the central $\mathrm{BH}$. Moreover, these outflows are not spherical, form in a geometrically thin layer which is located above the innermost part of the advective-disk, and have well defined direction of propagation.

Furthermore, the model may provide explanations for several additional issues related to the formation of jets and disks: 1) the $\mathrm{K} \alpha$ emission lines observed in Cyg X-1 which suggest that the disk-plasma in the vicinity of the last stable orbit is cold and rotates Keplerian, 2) The origin of the super-Keplerian motions observed in the Galactic black holes XTE J1650-500 and MCG-6-30-15 (Miller et al. 2002), which suggest that the central objects are spinning BHs, and 3) the origin of the extremely low mass-loaded jets observed in several AGNs and $\mu$-quasars.

\section{References}

Abramowicz, M., Igumenshchev, I., \& Lasota, J.-P. 1998, MNRAS, 293, 433

Balbus, S., \& Hawley, J. 1991, ApJ, 376 
Begelman, M. C., \& Chiueh, T. 1988, ApJ, 332, 872

Biretta, J., Junor, W., \& Livio, M. 2002, NewAR, 46, 239

Blandford, R., \& Znajek, R. 1977, MNRAS, 179, 433

Blandford, R., \& Payne, D. 1982, MNRAS, 199, 883

Blandford, R., \& Begelman, M. 1999, MNRAS, 303, L1

Blandford, R. 2001 [astro-ph/0110394]

Camenzind, M. 1990, RvMA, 3, 234

Camenzind, M. 2003, The Black hole environments, Les Houches Lecture 2002, Accretion Disks, Jets and high energy Phenomena in Astrophysics, ed. F. Menard et al. GDPS, in press

Di Matteo, T., Allen, S., Fabian, A., et al. 2003, ApJ, 582, 133

Enander, R. 1997, SIAM J. Sc. Comp., 18 (5), 1243

Esin, A., McClintock, J. E., Drake, J., et al. 2001, ApJ, 555, 483

Fendt, C., \& Memola, E. 2001, A\&A, 365, 631

Hawley, F., Gammie, C., \& Balbus, S. 1996, ApJ, 464, 690

Hawley, F., \& Balbus, S. 2002, ApJ, 573, 738

Heyvaerts, J., \& Norman, C. 1989, ApJ, 347, 1055

Hujeirat, A., \& Camenzind, M. 2000a, A\&A, 361, L53

Hujeirat, A., Camenzind, M. 2000b, A\&A, 362, L4

Hujeirat, A., \& Rannacher, R. 2001, NewAR, 45, 425

Hujeirat, A., Camenzind, M., \& Burkert, A. 2002a, A\&A, 386, 757

Hujeirat, A., Camenzind, M., \& Livio, M. 2002b, A\&A, 394, L9

Hujeirat, A. 2003, in preparation

Ghosh, P., \& Lamb, F. K. 1978, ApJ, 223, 83

Lazarian, A., \& Vishniac, E. 2000, RMxAC, 9, 55

Lovelace, R., Berk, H., \& Contopoulos, J. 1991, ApJ, 379, 696

Königl, A. 1989, ApJ, 342, 208

Koide, S., Shibata, K., Kudok, T., \& Meier, D. L. 2002, Science, 295, 1688
Miller, J. M., Fabian, A. C., \& Wijnands, R. 2002, ApJ, 570, L69

Mirabel, I. F. 2000 [astro-ph/0005591]

Mirabel, I. F. 2001, ApSS, 276, 153

Narayan, R., \& Yi, I. 1995, ApJ, 444, 231

Ogilvie, I., \& Livio, M. 2001, ApJ, 553, 158

Popham, R., \& Sunyaev, R. A. 2001, ApJ, 547, 355

Pudritz, R., \& Norman, C. 1983, ApJ, 274, 677

Paczyanski, B., \& Wiite, P. J. 1980, A\&A, 88, 23

Quataert, E. 1998, ApJ, 500, 978

Rees, M., Begelman, M., Blandford, R., \& Phinney, E. 1982, Nature, 295, 17

Rybicki, G., \& Lightman, A. P. 1979, Radiative processes (WileyInterscience Publication)

Shakura, N. I., \& Sunyaev, R. A. 1973, A\&A, 24, 337

Sandbæk, O., \& Leer, E. 1994, ApJ, 423, 500

Shu, F., Lizano, S., Ruden, S., \& Najita, J. 1988, ApJ, 328, L19

Shu, F., 1991, The Physics of Star Formation and Early stellar Evolution, ed. N. D. Kylafis, \& C. J. Lada (Dordrecht: Kluwer), 365

Shu, F., Najita, J., Ruden, S., \& Lizano, S. 1994, ApJ, 429, 781

Simon, T. 1999, ASP, 223, 235

Spiegel, E., \& Zahn, J.-P. 1992, A\&A, 265, 106

Stone, J. M., \& Pringle, J. E. 2000 [astro-ph/0009233]

Tout, C. A., \& Pringle, J. E. 1992, MNRAS, 259, 604

Turek, S. 1994, IWR preprint series, 70

Uchida, Y., \& Shibata, K. 1985, PASJ, 37, 515

Yuan, F., Markoff, S., \& Falke, H. 2002, A\&A, 383, 854 